\title{
A Perisynaptic Ménage à Trois between Dlg, DLin-7, and Metro Controls Proper Organization of Drosophila Synaptic Junctions
}

\author{
André Bachmann, ${ }^{1 \star}$ Oliver Kobler, ${ }^{2 *}$ Robert J. Kittel, ${ }^{3,4}$ Carolin Wichmann, ${ }^{5,6}$ Jimena Sierralta, $^{7}$ Stephan J. Sigrist, ${ }^{6}$ \\ Eckart D. Gundelfinger, ${ }^{2}$ Elisabeth Knust, ${ }^{1,8}$ and Ulrich Thomas ${ }^{2}$ \\ ${ }^{1}$ Institut für Genetik, Heinrich-Heine-Universität Düsseldorf, D-40225 Düsseldorf, Germany, ${ }^{2}$ Leibniz Institute for Neurobiology, Department of \\ Neurochemistry, D-39118 Magdeburg, Germany, ${ }^{3}$ Institut für Physiologie, Universität Würzburg, D-97070 Würzburg, Germany, ${ }^{4}$ Rudolf Virchow Deutsche \\ Forschungsgemeinschaft Research Center for Experimental Medicine, D-97078 Würzburg, Germany, ${ }^{5}$ European Neuroscience Institute, D-37077 \\ Göttingen, Germany, ${ }^{6}$ Institut für Biologie, Freie Universität Berlin, 14195 Berlin, Germany, ${ }^{7}$ Instituto de Ciencias Biomédicas, Facultad de Medicina, \\ Universidad de Chile, Santiago 6530499, Chile, and ${ }^{8}$ Max Planck Institute of Cell Biology and Genetics, D-01307 Dresden, Germany
}

Structural plasticity of synaptic junctions is a prerequisite to achieve and modulate connectivity within nervous systems, e.g., during learning and memory formation. It demands adequate backup systems that allow remodeling while retaining sufficient stability to prevent unwanted synaptic disintegration. The strength of submembranous scaffold complexes, which are fundamental to the architecture of synaptic junctions, likely constitutes a crucial determinant of synaptic stability. Postsynaptic density protein-95 (PSD-95)/ Discs-large (Dlg)-like membrane-associated guanylate kinases (DLG-MAGUKs) are principal scaffold proteins at both vertebrate and invertebrate synapses. At Drosophila larval glutamatergic neuromuscular junctions (NMJs) DlgA and DlgS97 exert pleiotropic functions, probably reflecting a few known and a number of yet-unknown binding partners. In this study we have identified Metro, a novel p55/MPP-like Drosophila MAGUK as a major binding partner of perisynaptic DlgS97 at larval NMJs. Based on homotypic LIN-2,-7 (L27) domain interactions, Metro stabilizes junctional DlgS97 in a complex with the highly conserved adaptor protein $D$ Lin-7. In a remarkably interdependent manner, Metro and DLin-7 act downstream of DlgS97 to control NMJ expansion and proper establishment of synaptic boutons. Using quantitative 3D-imaging we further demonstrate that the complex controls the size of postsynaptic glutamate receptor fields. Our findings accentuate the importance of perisynaptic scaffold complexes for synaptic stabilization and organization.

\section{Introduction}

The establishment of neural networks involves mechanisms that coordinate the assembly and selective stabilization of synapses (Goda and Davis, 2003). Multivalent scaffold molecules that link transmembrane proteins to the cytoskeleton are candidate determinants of synapse stability. Recent studies imply a stabilizing role for Postsynaptic density protein-95 (PSD-95), a principal vertebrate synaptic scaffold protein, during activity-dependent maturation of glutamatergic synapses (Ehrlich et al., 2007; Steiner et al., 2008; Kim et al., 2009). The linkage of PSD-95 to ionotropic glutamate receptors (GluRs), however, makes it diffi-

Received Feb. 12, 2010; accepted March 1, 2010.

This work was supported by the Deutsche Forschungsgemeinschaft (DFG-Sonderforschungsbereich 854), the Leibniz Gemeinschaft (WGL-Pakt für Forschung und Innovation), and a Max Planck award from the Alexander von Humboldt Foundation and the Max Planck Society to E.D.G. R.J.K. is supported by the Deutsche Forschungsgemeinschaft Emmy-Noether Program. We thank Dr. Martin Heine for helpful discussion and Ilona Linke for technical assistance. We are grateful to Jan Pielage for $\beta$-spectrin RNAi lines. We thank the Bellen group, the stock centers at Bloomington and Szeged, the Exelixis team at Harvard Medical School, and the Developmental Studies Hybridoma Bank (lowa) for nonprofit provision of fly stocks and monoclonal antibodies.

${ }^{*}$ A.B. and $0 . K$. contributed equally to this work.

Correspondence should be addressed to Ulrich Thomas, Department of Neurochemistry, Leibniz Institute for Neurobiology, Brenneckestrasse 6, D-39118 Magdeburg, Germany. E-mail: thomas@ifn-magdeburg.de.

DOI:10.1523/JNEUROSCI.0778-10.2010

Copyright $\odot 2010$ the authors $\quad 0270-6474 / 10 / 305811-14 \$ 15.00 / 0$ cult to assess a direct involvement in synaptic structural integrity independent from activity-related effects. Moreover, the existence of additional PSD-95/Discs-large (Dlg)-like membraneassociated guanylate kinases (DLG-MAGUKs) accounts for partial redundancy (Elias et al., 2006) and functional diversification including perisynaptic and extrasynaptic activities (Kim et al., 1996; Sans et al., 2001; Chetkovich et al., 2002; Nakagawa et al., 2004; Schlüter et al., 2006; Waites et al., 2009).

In Drosophila a single gene, discs-large ( $\mathrm{dlg}$ ), encodes DlgA and DlgS97. The latter is specified by an N-terminal L27 domain (Mendoza et al., 2003) and thus corresponds to the predominant isoform of vertebrate SAP97 (Schlüter et al., 2006). Both isoforms, collectively referred to as Dlg, are present at glutamatergic larval neuromuscular junctions (NMJs) (Ataman et al., 2006). Strikingly, Dlg omits GluR-containing PSDs but is enriched within the subsynaptic reticulum (SSR), a postsynaptic membrane specialization commonly categorized as perisynaptic (Prokop, 2006). Strong dlg mutants display aberrant motornerve terminal morphology and severely reduced SSR complexity (Ataman et al., 2006; Gorczyca et al., 2007). Dlg further controls the size of synaptic contacts (i.e., active zones and PSDs) (Karunanithi et al., 2002; Chen and Featherstone, 2005), possibly involving the perisynaptic cell adhesion mol- 
ecule Fasciclin II (FasII) as binding partner (Ataman et al., 2006). Similar to various $d l g$ alleles, strong fasII alleles display enlarged active zones (Stewart et al., 1996). Mutations that specifically abolish DlgS97, however, result in a similar phenotype while leaving junctional FasII largely unaffected (Mendoza-Topaz et al., 2008), suggesting that DlgS97 acts in a FasII-independent pathway to restrict synaptic contacts.

To specify the role of DlgS97 we have previously begun to analyze isoform-specific interactions and shown that DlgS97 is crucial for proper NMJ localization of the PDZ domain protein DLin-7. We further predicted this interaction to rely on a linker protein expressed in muscles but not in epithelia (Bachmann et al., 2004). Proteins bearing a tandem of L27 domains such as the MAGUK CASK/mLin-2 or members of the p55 subfamily of MAGUKs have emerged as primary candidates to serve the linkage between vertebrate Lin-7 (Veli) and SAP97 in epithelial cells (Karnak et al., 2002; Lee et al., 2002; Bohl et al., 2007). Here, we introduce Metro, a novel Drosophila MAGUK, as the missing link between DlgS97 and DLin-7 at NMJs. Genetic analyses reveal that the three scaffold proteins control each other. NMJs lacking Metro display reduced growth and are predestined to structural abnormalities. Notably, Metro and DLin-7 are involved in the dimensioning of glutamate receptor fields. Our findings show that Metro and DLin-7 augment the complexity of the perisynaptic scaffold system and thereby control the synaptic organization of the NMJ.

\section{Materials and Methods}

Flies. Stocks and crosses were grown at $25^{\circ} \mathrm{C}$ and at similar population densities. Canton $S$ was used as the wild-type strain. Balancer chromosomes $\mathrm{CyO}^{\text {actGFP }}$ and TM6 were used to distinguish homozygous or hemizygous mutant larvae from heterozygous siblings. The following strains have been described previously: Gal4-OK6 (Landgraf et al., 2003), Gal4-C57 (Thomas et al., 2000), $d^{\text {Sg97-138 }}$ (Mendoza-Topaz et al., 2008), dLin-7 $^{\# 66}$ (Bachmann et al., 2008b), UAS-Flag-DLin-7 (Bachmann et al., 2004), $\mathrm{cmg}^{\mathrm{X} 307}$ (Lu et al., 2003), and $\beta$-spectrin-specific RNAi effector lines (Pielage et al., 2006). The tubP-Gal4-line (Lee and Luo, 1999), deficiency line $D f(2 R) E 3363$, and the $C G 30021^{B G 02148}$ gene trap line (Lukacsovich et al., 2001; Bellen et al., 2004) were obtained from the Bloomington stock center. The isoform-specific allele $d l^{A-51.1}$ and the metro $^{* 47}$ mutant resulted from imprecise excision of P-elements by using a genomic transposase source (Robertson et al., 1988). The $d l^{S 97-f l p V}$ allele was generated by FRT (Flp recognition target)-specific recombination in transheterozygous $d l^{X P-d 08753} / \mathrm{dlg}^{R B-e 03622}$ females upon repeated heat shock-induced expression of FLP recombinase (Parks et al., 2004). The deletion $D f(3 R) D L i n-7^{20 l}$ was generated by mobilization of the P-element in the CG11791 KG00327.

Transgenic constructs and germline transformation. The EST-clone GH12103 (BDGP) represents a full-length metro cDNA that was used for all subsequent cloning procedures. A UAS-metro construct was generated by introducing a PCR-amplified metro cDNA (primers: GH12103-5, CTTGTAAAGAATTCGCAAGGACAG; GH12103-3; CTTCTCCGTTTCTCGAGCAGATTG) into pUAST (Brand and Perrimon, 1993). The construct for metro-specific DNA-mediated RNA interference was generated as follows: a fragment covering the L27 and PDZ domains was PCR amplified (primers: GH12103-5, see above; GH12103-RNAi-3, TTTGGACTCGAGCTGGGCG), subcloned into pBsc $\mathrm{KS}^{+}$, excised with BamHI and Asp718, and introduced into pHIBS followed by cloning into pUAST as described previously (Nagel et al., 2002). Only sequence-controlled PCR fragments were used for cloning. Transgenic flies were generated by P-element-mediated germ line transformation (Bachmann and Knust, 2008).

Transcript analyses. In situ hybridizations and Northern blot analyses were performed following standard procedures using a digoxigeninlabeled RNA antisense probe that recognizes the full-length metro cDNA. For RT-PCR total RNA from 20 body walls of third-instar larvae was extracted using the NucleoSpin RNA II kit from Macherey-Nagel. Five hundred nanograms of total RNA were used as template (OneStep RT-PCR Kit, Qiagen). The following primer pair was used for detection of metro transcripts: Act2-Metro-5, CTGTCGGAATTCACGAGGACG; GH12103-3, see above. As an internal control transcripts from the rp49 gene were detected using primers rp49-5, AGATCGTGAAGAAGCGCACC; and rp49-3, CGATCCGTAACCGATGTTGG.

Antibodies and immunocytochemistry. Antisera against Metro were generated by repetitive immunization of a rat with an affinity-purified GST-Metro fusion protein covering the PDZ and SH3 domains (Glu ${ }^{134}$ to Leu ${ }^{310}$ ). Larval body wall preparations were performed according to the protocol of Bellen and Budnik (2000). Samples were fixed for $10 \mathrm{~min}$ in Bouin's fixative for stainings involving rat anti-Metro (1:150). Otherwise, samples were fixed in $4 \%$ paraformaldehyde for $20 \mathrm{~min}$. Upon fixation samples designated for direct comparison were coincubated during all subsequent steps with small incision marks allowing for an unambiguous tracing of genotypes. All wash steps and antibody incubations were performed in phosphate buffer, $\mathrm{pH} 7.2$, containing $0.2 \%$ Triton X-100. Previously described antibodies were used as follows: rabbit antisera anti-DLin-7 1:1000 (Bachmann et al., 2004), anti-Dlg $\operatorname{Sg7N~}_{\text {N }} 1: 1000$ (Mendoza et al., 2003), anti-GluRIID 1:500 (Qin et al., 2005), and mono-

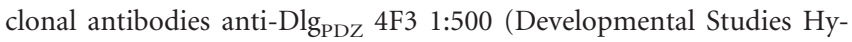
bridoma Bank), anti-FasII 1D4 1:10 (Developmental Studies Hybridoma Bank), and anti-Brp NC82 1:100 (Wagh et al., 2006). Cy3- or Cy5conjugated goat anti-HRP antibodies and secondary antibodies coupled with Cy3 (Jackson ImmunoResearch Laboratories), Alexa-488, or Alexa568 (Invitrogen) were used at 1:200.

All figures were processed using ImageJ or Adobe Photoshop 7.0.

Image acquisition, $3 D$ reconstructions, and quantitative image analysis. Confocal images were acquired on a Leica TCS SP2-AOBS scanning system. All quantitative analyses were performed on muscle 6/7 NMJs of abdominal segment A2.

Fluorescence intensities were measured on maximum projections of confocal stacks (step size $0.75 \mu \mathrm{m}$ ) taken with a $63 \times 1.4 \mathrm{NA}$ oil objective with the same settings for all samples and with genotypes alternating within a given session. Bleaching was minimized by scanning at $400 \mathrm{~Hz}$ with a format of $512 \times 512$ pixels combined with a zoom factor of 3 to obtain reasonable resolution. A mask outlining type Ib boutons was created in ImageJ (Rasband, 1997-2009) based on the Otsu threshold function. Integrated intensities of at least two branches per NMJ were determined and divided by the summed area of their regions of interest (ROIs).

For counting of Brp-positive active zones we acquired stacks of complete NMJs with a $40 \times 1.25$ NA oil objective and settings that yielded a voxel size of $130 \times 130 \times 285 \mathrm{~nm}$. Stacks were subjected to a $3 \mathrm{D}$-blind deconvolution algorithm implemented in AutoDeblur X2 (Media Cybernetics) software. Ten iterations were applied. Resulting image stacks were normalized by a linear histogram stretch and 3D-rendered in Imaris 6 (Bitplane AG, Zurich, Switzerland). The Imaris spot detection wizard was used to identify and count all Brp-stained sites based on the same threshold for all images.

For the analysis of receptor fields samples were scanned using a $63 \times$ 1.32 NA oil objective and a format of $512 \times 512$ pixels at $400 \mathrm{~Hz}$. Combined with zoom factor 6 a voxel size of $78 \times 78 \times 163 \mathrm{~nm}$ was obtained. Blind 3D deconvolution was performed with 20 iteration steps and image stacks were normalized and 3D rendered. Analysis was restricted to GluR clusters within the NMJ as defined by a slightly oversized mask based on the HRP costaining, which allowed exclusion of extrajunctional clusters. For segmentation of receptor fields we applied the surface wizard of Imaris 6, which generates surfaces according to an intensity-based threshold. Local maxima (seeds) within each surface provided starting points for a region-growing algorithm, which led to the separation of individual receptor fields. A seed minimum diameter of $0.4 \mu \mathrm{m}$ was used throughout as it was found to yield most reliable representations of GluR fields in the wild type. Segmented areas of $<0.3 \mu \mathrm{m}^{2}$ were excluded as they almost exclusively represented extrasynaptic clusters within the NMJ mask. The mean surface areas determined in this way correspond reasonably well to previously published diameters of active zones if we 
consider them as either spherical or as flat cylinders with a height of $100-150 \mathrm{~nm}$.

The size of boutons was measured using ImageJ, based on high-resolution confocal stacks of anti-HRP and anti-Brp-stained NMJ branches. In brief, maximum projections were subjected to a median filter (radius 2) and then converted to a mask, yielding a binary image. This was used to create an ROI, which was subdivided into individual boutons by using the brush tool. Next to the morphology the absence of Brp staining in between boutons served as a reliable criterion for segmentation.

All statistical analyses were performed using GraphPad Prism (version 4) software.

Quantification of bouton numbers. Low-resolution confocal images of anti-Dlg-stained NMJs were cataloged in scrambled order and the projection area for each pair of muscles 6/7 was determined using ImageJ. Counting of boutons was then performed based on both HRP- and Dlgspecific immunofluorescence using a $63 \times$ oil objective on an epifluorescence microscope and with the tester blinded for the genotypes of samples. Each value was assorted to the respective, easily identifiable NMJ within the catalog. Genotypes were revealed and entered in the catalog by the end of each session.

Pull-down assay, immunoprecipitations, and Western blot analysis. Biotinylated Dlg-S97, DLin-7, and Metro proteins were generated by in vitro transcription/translation with the TNT coupled reticulocyte lysate system/nonradioactive translation detection system (Promega) and incubated with equal amounts of glutathione Sepharose 4B (GE Healthcare)-coupled, bacterially induced GST fusion proteins and GST in pull-down buffer (20 mм Tris, pH 8, $0.2 \mathrm{M}$ EDTA, $0.1 \mathrm{M} \mathrm{NaCl}, 1 \mathrm{~mm}$ DTT, $0.2 \%$ IGEPAL, $1 \mu \mathrm{M}$ Pefabloc, $5 \mu \mathrm{M}$ leupeptin, $1 \mu \mathrm{M}$ pepstatin, 0.3 $\mu \mathrm{M}$ aprotinin) overnight at $4^{\circ} \mathrm{C}$. Beads were pelleted, washed four times in pull-down buffer, and eluted by boiling in SDS-PAGE sample buffer. Eluted proteins were analyzed by SDS-PAGE and detected with streptavidin-HRP (Roche, 1:1000). The following GST fusion proteins were used: GST-Metro ${ }^{\text {L27N }}$ (primers GH12103-L27N5, GTTGTGTGAATTCCTAGAAGCAG; GH12103-L27N3, CCAGGACCTCGAGCAACACC), GST-Metro ${ }^{\text {L27N+C }}$ (primers GH12103-L27N5, see above; GH12103-L27C3, GCACAATCTCGAGGGTCTCC), GST-DLin-7 ${ }^{\text {L27 }}$ (primers: DLin-7-L27-5, GGCCGAATTCGCAGAACCACTG; DLin7-L27-3, GGGCAGGTCGACGACTCTGGG), and GST-DLin-7 ${ }^{\text {PDZ }}$ (primers DLin-7-PDZ-5, CACTGTGGCAGAATTCGCAGC; DLin-7PDZ-3, GAACTAGTCGACCTGTATGGTC). GST-Dlg-S97N comprises $\mathrm{Met}^{1}$ to Ile ${ }^{150}$ of DlgS97 encoded by a $0.45 \mathrm{~kb}$ BglII fragment inserted into pGEX-2T. Immunoprecipitation experiments from larvae were performed at $4^{\circ} \mathrm{C}$ : wild-type body walls from 35 dissected larvae were homogenized in $500 \mu$ l of lysis buffer containing $20 \mathrm{~mm}$ Tris- $\mathrm{HCl}, \mathrm{pH} 8$, $150 \mathrm{~mm} \mathrm{NaCl}, 10 \%$ glycerol, 2 mм EDTA, 10 mм CHAPS (3-[(3cholamidopropyl)dimethylammonio]-1-propanesulfonate), and protease inhibitors ( $1 \mu \mathrm{M}$ Pefabloc, $5 \mu \mathrm{M}$ leupeptin, $1 \mu \mathrm{M}$ pepstatin, $0.3 \mu \mathrm{M}$ aprotinin). Following a $2 \mathrm{~h}$ extraction on a shaker, homogenates were cleared from cuticle debris by centrifugation for $5 \mathrm{~min}$ at $3000 \times \mathrm{g}$. One hundred forty microliters of the supernatant were loaded with $260 \mu \mathrm{l}$ of lysis buffer onto $30 \mu \mathrm{l}$ of Protein G Sepharose (Pharmacia Biotech) and preincubated overnight at $4^{\circ} \mathrm{C}$ on a shaker. After an incubation for $2 \mathrm{~h}$ with $2 \mu \mathrm{l}$ each of anti-DLin-7 antibody and control antibody, $30 \mu \mathrm{l}$ of Protein G Sepharose was added, followed by an overnight incubation at $4^{\circ} \mathrm{C}$ on a shaker. The Protein G Sepharose precipitate was washed four times in lysis buffer, supplied with $20 \mu \mathrm{l}$ of $2 \times$ SDS sample buffer, and boiled for $5 \mathrm{~min}$. Equivalents of $\sim 1$ body wall (input controls) or 3 body walls (precipitates) were separated by SDS-PAGE and blotted onto nitrocellulose transfer membrane. Upon blockage in TBST/5\% dry milk, the membrane was incubated overnight with rat anti-Metro antibody at 1:1000. For comparative Western blot analyses, 10-12 dissected body walls of each genotype were frozen at $-70^{\circ} \mathrm{C}$ and homogenized in $60 \mu \mathrm{l}$ of radioimmunoprecipitation assay buffer containing $0.1 \mathrm{M}$ DTT and a protease inhibitor mixture (Complete, Roche Applied Science). Homogenates were cleared by centrifugation for $5 \mathrm{~min}$ at $3000 \mathrm{rpm}$ at $4^{\circ} \mathrm{C}$ and supernatants were supplemented with $20 \mu \mathrm{l}$ of $4 \times$ SDS sample buffer. Upon 5 min of boiling equivalents of $\sim 1$ body wall were separated by SDSPAGE and blotted. Primary antibodies were applied overnight at $4^{\circ} \mathrm{C}$
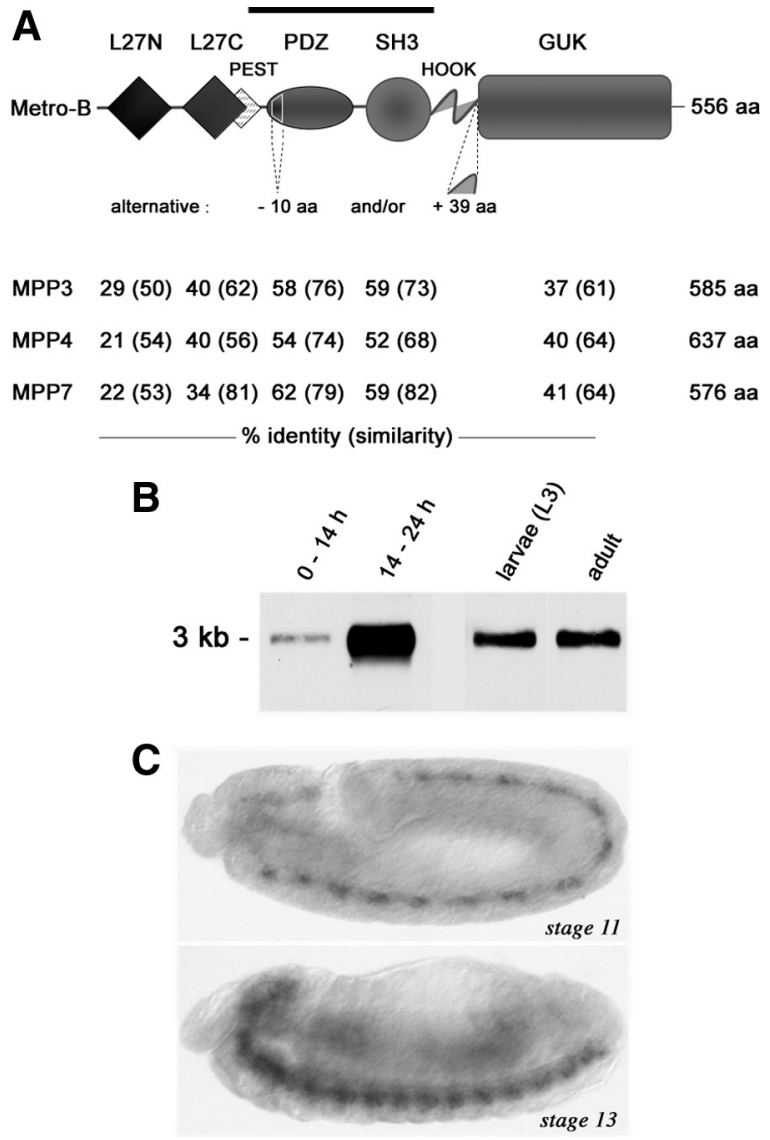

Figure 1. Metro is a neural MPP-like MAGUK. A, Domain organization of Metro. The degree of conservation in human MPP3, MPP4, and MPP7 is listed beneath. Splice variations may affect the PDZ and HOOK domains as indicated. The PEST sequence is conserved in MPP3 and MPP7. A bar indicates the region used for generating antibodies. $\boldsymbol{B}$, Northern blot analysis on $\mathrm{mRNA}$ of $0-14 \mathrm{~h}$ and $14-24 \mathrm{~h}$ embryos, third-instar larvae, and adults. C, In situ hybridization on stage 11 and stage 13 embryos (lateral view, anterior left) reveals metro mRNA in the developing CNS.

at the following concentrations: rabbit anti- $D$ Lin-7 1:2000, rabbit anti-Dlg $_{\text {S97N }} 1: 4000$, rabbit anti-Metro 1:1000, rat anti-Metro 1:1000, monoclonal anti-Dlg $\lg _{\mathrm{PDZ}} 1: 2000$, and monoclonal anti- $\beta$-tubulin E7 1:500 (Developmental Studies Hybridoma Bank). Peroxidase-conjugated secondary antibodies in combination with the ECL system (GE Healthcare Pharmacia Biotech) were used to detect immunoreactive bands.

Electron microscopy. Transmission micrographs were obtained from muscles 6/7 from dissected third-instar larvae as described by Wagh et al. (2006).

Electrophysiology. Two-electrode voltage-clamp recordings were performed essentially as previously described (Kittel et al., 2006). In brief, two intracellular electrodes (8-17 MOhm) were used to clamp muscle 6 (segment A2 or 3; input resistance $>5 \mathrm{MOhm}$, initial $V_{\mathrm{m}}-52$ to -70 $\mathrm{mV}$ ) of late-third-instar male larvae (raised at $25^{\circ} \mathrm{C}$ ) and to record both minis $\left(V_{\text {hold }}-80 \mathrm{mV}\right)$ and eEJCs $\left(V_{\text {hold }}-60 \mathrm{mV}\right)$ at room temperature in HL-3 solution (Stewart et al., 1994) containing $1 \mathrm{~mm} \mathrm{Ca}^{2+}$.

\section{Results}

\section{Identification of Metro, a p55/MPP-like MAGUK that binds DlgS97 and DLin-7}

To identify Drosophila proteins, which carry two L27 domains and hence might interact with DlgS97 and DLin-7 simultaneously, we used sequences of L27 domain proteins for BLAST search screening at www.flybase.org. Four MAGUKs were found to meet this criterion. Stardust (Sdt), a homolog of PALS-1/ MPP5, interacts with DLin-7 at epithelial membranes but not at NMJs (Bachmann et al., 2004). Similarly, Varicose (Vari), an 
MPP2-like protein (Wu et al., 2007; Bachmann et al., 2008a; Moyer and Jacobs, 2008), binds to both DlgS97 and DLin-7 in vitro but does not colocalize with them at NMJs (A. Bachmann and U. Thomas, unpublished data). Thus, the CASK homolog Camguk (Cmg) and predicted translational products of a gene annotated as CG30021 (CG30021-P) remained as candidate linker proteins. Regarding its involvement in a ménage à trois with DlgS97 and DLin-7 (see below) we propose to refer to CG30021-P as "Metro" (see supplemental Note, available at www. jneurosci.org as supplemental material).

All predicted Metro isoforms comprise two L27 domains followed by one PDZ, one $\mathrm{SH} 3$, and one carboxy terminal GUK domain (Fig. 1A). This domain organization is typical for p55/MPP-like MAGUKs (Caruana, 2002), among which the vertebrate members MPP3, MPP4, and MPP7 share highest similarity with Metro (Fig. $1 A)$. Northern blot analyses on mRNA from embryos, third-instar larvae, and adult flies revealed a single metro-specific band of $3 \mathrm{~kb}$ (Fig. $1 \mathrm{~B}$ ). As this assay may fail to resolve subtle splice variations, we conducted RT-PCR on mRNA from third-instar larval body walls. Using primers designed to amplify the complete coding region, we repeatedly detected just one cDNA encoding a 556 aa protein (Fig. $1 A$ ). We hence consider this variant as the prevailing muscle isoform and refer to it as Metro-B in accordance to its annotation as variant B in Flybase. In situ hybridizations revealed that during embryogenesis metro is mainly expressed in the developing CNS, in which it is detectable from around stage 11 onwards (Fig. $1 C)$. Both the temporal and spatial expression patterns of metro mRNA are consistent with a role for Metro as a neural scaffold protein.

We next evaluated Metro for physical interaction with DlgS97 and DLin-7 by monitoring the binding of in vitro-translated biotinylated protein samples to immobilized GST fusion proteins. Biotinylated $D$ Lin-7 was efficiently pulled down by an immobilized GST fusion protein comprising both L27 domains of Metro but not by either GST alone or a fusion protein lacking the C-terminal L27 domain (Fig. 2A). The reverse experiment confirmed that Metro binds to the L27 domain of $D$ Lin-7 but not to its PDZ domain (Fig. 2 B). Fusion proteins comprising both L27 domains or the N-terminal L27 domain of Metro alone were found to pull down the N-terminal domain of DlgS97 (Fig. 2C). Consistently, biotinylated full-length Metro was bound by immobilized GST-DlgS97N but not by GST (Fig. 2D).

\section{Metro forms a postsynaptic protein complex with DlgS97} and DLin-7

For the detection of Metro, we generated an antiserum against its PDZ and SH3 domains (Fig. 1A). Using this antiserum for West-
E
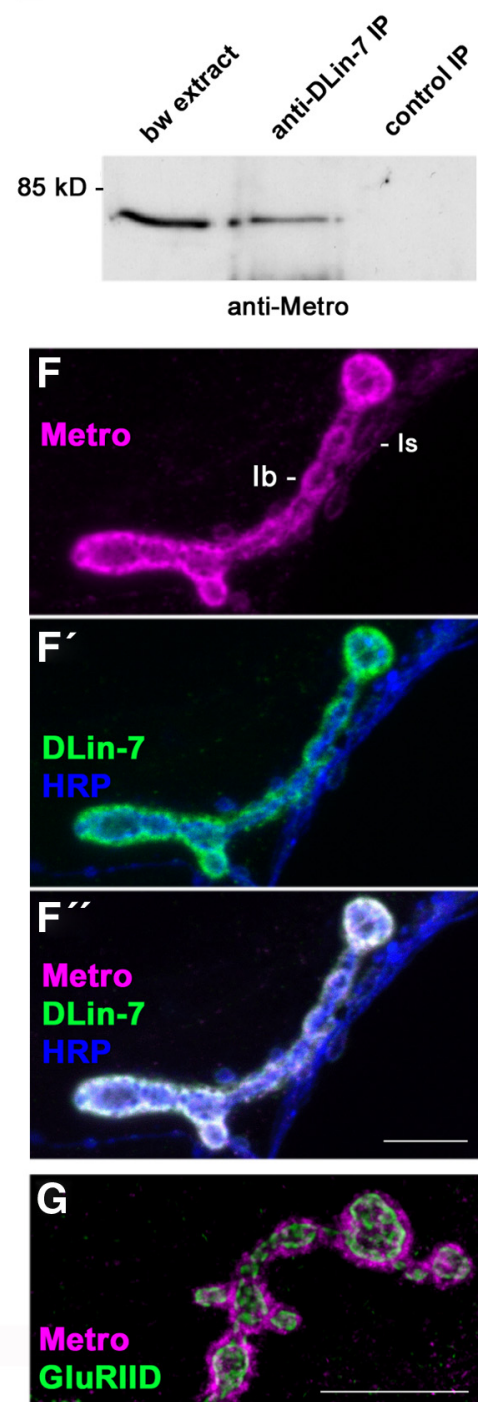

$-85 k D$

Figure 2. DlgS97, Metro, and DLin-7 interact in vitro and at NMJs. A-D, Pull-down experiments. Input controls (left lanes) and fractions bound to immobilized GST fusion proteins were analyzed by Western blot analysis using streptavidin-peroxidase conjuwas used as a control. $\boldsymbol{F}-\mathbf{G}$, Maximum projections of confocal stacks displaying the localization of Metro relative to DLin-7, HRP epitope $\left(\boldsymbol{F}-\boldsymbol{F}^{\prime \prime}\right)$ and glutamate receptors $(\boldsymbol{G})$ at NMJs of muscle $12\left(\boldsymbol{F}-\boldsymbol{F}^{\prime \prime}\right)$ or $4(\boldsymbol{G})$. Scale bars: $10 \mu \mathrm{m}$.

ern blot analysis we detected a Metro-specific $70 \mathrm{kDa}$ band in body wall extracts (Figs. $2 E, 3 B$ ). Notably, the same band was detected in anti- $D$ Lin-7 immunoprecipitates from body wall extracts but not in control samples (Fig. $2 E$ ), implying that the two proteins are linked in vivo. Immunofluorescence analyses on body wall muscles revealed prominent Metro-specific labeling at type Ib boutons of NMJs and a weak staining of type Is boutons (Fig. 2 F). Just like Dlg (Bachmann et al., 2004), Metro perfectly colocalizes with DLin-7 at NMJs and triple stainings including anti-HRP as a neuronal surface marker showed that both proteins are enriched postsynaptically (Fig. $2 F-F^{\prime \prime}$ ). The postsynaptic compartment around type I boutons typically harbors synaptic glutamate receptor fields and the perisynaptic SSR. Metro, like Dlg and DLin-7, is confined to the SSR (Fig. 2G). Together, these findings strongly suggest that Metro links DlgS97 and DLin-7 via L27 domain interactions (Fig. S2, available at www.jneurosci.org as supplemental material) within the perisynaptic compartment of glutamatergic NMJs. 

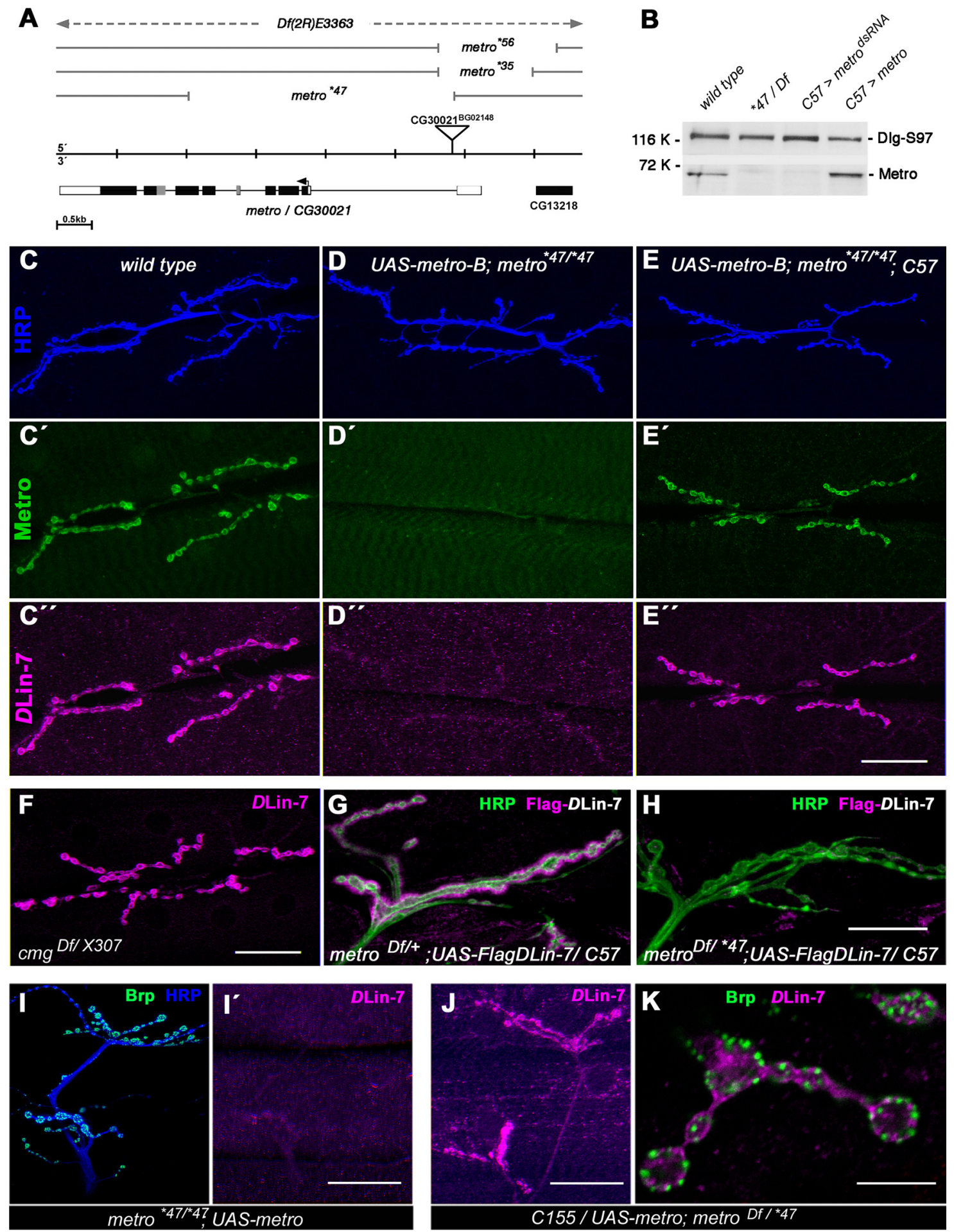

Figure 3. Loss of metro abolishes DLin-7 at NMJs. A, Exon-intron structure of metro and mapping of mutant alleles. Open and filled boxes represent untranslated and coding regions, respectively. Imprecise excision of the P-element $B G 02148$ yielded hypomorphic alleles $(* 35, * 56)$ and the null allele $* 47$, a deletion lacking exons $2-5$ and part of exon 6 . Df (2R)E3363 uncovers metro completely. $B$, Western blot analysis of body wall extracts from the wild type, metro mutants ( $\left.{ }^{*} 47 / D f\right)$, muscle-specific RNAi (UAS-metro ${ }^{d s N A A}$; Gal4 - C57), and overexpression (UAS-metro- $B ; ;$ Gal4 - C57). The same blot was probed for DlgS97 (120 kDa) and Metro (70 kDa). C $-\boldsymbol{E}^{\prime \prime}$, NMJs at muscles $6 / 7$ of the wild type, metro mutants, and metro mutants expressing a metro- $B$ transgene in muscles, stained for HRP $(\boldsymbol{C}-\boldsymbol{E})$, Metro $\left(\boldsymbol{C}^{\prime}-\boldsymbol{E}^{\prime}\right)$, and $D$ Lin-7 $\left(\boldsymbol{C}^{\prime \prime}-\boldsymbol{E}^{\prime \prime}\right)$. $\boldsymbol{F}, \boldsymbol{D L}$ Lin-7 appears unaffected at $c m g$ mutant NMJs. $\boldsymbol{G}, \boldsymbol{H}$, Flag-tagged $D$ Lin-7 localized to type I boutons at muscle 12 in the presence $(\boldsymbol{G})$ but not in the absence $(\boldsymbol{H})$ of Metro. $\boldsymbol{I}-\boldsymbol{K}$, Presynaptic $D$ Lin-7 visualized at muscles $12 / 13$ upon neuron-specific expression of UAS-metro-B in metro mutants $(\boldsymbol{J})$; controls lacking the Gal4 activator $\left(\boldsymbol{I}, \boldsymbol{I}^{\prime}\right)$; $D$ Lin-7 localizes next to Brp-labeled active zones (K). Scale bars: (in $\boldsymbol{E}^{\prime \prime}, \boldsymbol{F}, \boldsymbol{I}^{\prime}$, and $\left.\boldsymbol{J}\right) \boldsymbol{C}-\boldsymbol{F}, \boldsymbol{I} \boldsymbol{I}, \mathbf{J}, 40 \mu \mathrm{m}$; (in $\left.\boldsymbol{H}\right) \boldsymbol{G}, \boldsymbol{H}, 20 \mu \mathrm{m} ; \boldsymbol{K}, 10 \mu \mathrm{m}$.

Metro is essential for the recruitment of $D$ Lin-7 to NMJs

For further studies on the role of Metro we generated transgenic flies and mutants for targeted expression and suppression of the protein, respectively. Mutants were obtained by imprecise exci- sion of a P-element inserted into the first intron of metro (Fig. $3 A$ ). One excision line, metro $^{*}{ }^{* 7}$, emerged as a null allele (Fig. $3 A$ ), which is homozygous viable and fertile. Western blot analyses confirmed the absence of Metro in both hemizygous 
and homozygous metro ${ }^{* 77}$ body wall extracts (Fig. 3B) (data not shown). Musclespecific expression of a metro-directed dsRNA by means of the activator strain Gal4-C57 reduced Metro to virtually the same extent (Fig. 3B), indicating that Metro is largely derived from muscles rather than from associated epithelial tissues. Moreover, Gal4-C57-induced expression of a UAS-metro-B construct (Fig. $3 B$ ) fully restored Metro-specific labeling at NMJs in the absence of endogenous Metro (Fig. 3C- $E^{\prime}$ ).

Both Metro (this work) and Cmg (Lee et al., 2002; Bachmann et al., 2004) bind to DlgS97 and DLin-7 in vitro. We thus tested whether either Metro or Cmg alone or a combination of both accounts for the NMJ localization of $D$ Lin-7 (Fig. $3 C^{\prime \prime}-F$ ). Strikingly, DLin-7-specific labeling was reduced to background levels at metro mutant NMJs (Fig. 3 $3 D^{\prime \prime}$ ), whereas mutants lacking intact $\mathrm{Cmg}$ displayed no discernible effect on DLin-7 (Fig. $3 F$ ). Moreover, muscle expression of Metro-B was sufficient to restore wild-type levels of junctional DLin-7 in the metro mutant background (Fig. $3 E^{\prime \prime}$ ). Consistent with the virtual absence of Metro in epithelia, the apical localization of DLin-7 in wing imaginal disc epithelia remains unaffected in metro mutants (Fig. S1 $A, B$, available at www.jneurosci.org as supplemental material). Less expectedly, enrichment of $D$ Lin-7 in the neuropil of the larval CNS also appeared unaffected (Fig. S1C-E, available at www.jneurosci.org as supplemental material). Western blot analysis showed that loss of Metro causes an appreciable reduction of $D$ Lin-7 within body wall extracts (see Fig. $8 H$ ). To rule out that reduced expression levels account for the lack of DLin-7 at metro mutant NMJs, we used Gal4-C57 to drive expression of Flag-tagged $D$ Lin-7 in the presence or absence of Metro. Despite high expression levels, Flag- $D$ Lin-7 localized at NMJs only in the presence of Metro (Fig. 3G,H). We therefore conclude that the physical interaction between Metro and DLin-7 is essential for postsynaptic localization of DLin-7.

Strong labeling of DLin-7 within the postsynaptic SSR may preclude its unequivocal detection at the presynaptic membrane. Targeted expression of Metro-B in motorneurons of metro mutants, however, allowed us to detect endogenous $D$ Lin-7 in juxtaposition to the active zone marker protein Bruchpilot (Brp) (Wagh et al., 2006) (Fig. 3I-K). Metro is thus able to recruit DLin-7 to perisynaptic membrane compartments on both the presynaptic and postsynaptic side of NMJs.

\section{New $d l g$ alleles reveal a dual mode of \\ Metro-DLin-7 recruitment}

We have previously shown that DlgS97 rather than DlgA promotes the NMJ localization of DLin-7 (Bachmann et al., 2004). Thus, if Metro is the proposed linker protein, then its junctional anchorage should also depend on DlgS97. To evaluate this prediction, we generated $d l g$-alleles $A-51.1$ and S97-flpV lacking expression of either DlgA or DlgS97 (Fig. 4A, B; Fig. S3A, available at www.jneurosci.org as supplemental material). Compared with wild-type NMJs, lack of DlgA in $d l^{A-51.1}$ mutants caused a moderately increased level of junctional Metro (Fig. $4 C, D$ ). This is consistent with DlgS97 compensating for loss of DlgA, thus leaving the overall amount of Dlg at NMJs unchanged (Fig. S3 B, C, available at www.jneurosci.org as supplemental material). Unexpectedly, $d l^{S 97-f l p V}$ mutant NMJs, which lack DlgS97 but retain DlgA (Fig. 4A, B; Fig. S3 D, E, available at www.jneurosci.org as supplemental material), displayed readily detectable albeit clearly reduced levels of junctional Metro (Fig. $4 E$ ) and DLin-7 (Fig. $\left.4 F, G^{\prime}\right)$. Immunofluorescence measurements revealed a $76.3 \%$ reduction of $D$ Lin-7 at $d l g^{S 97-f l p V}$ versus wild-type NMJs, whereas anti-Dlg $\operatorname{PDZ}_{\mathrm{PDZ}}$ signal intensity was reduced by only $26.3 \%$ (Fig. $4 \mathrm{H}$ ). The disproportionate reduction of DLin-7 confirms that DlgS97 has a strong, isoform-specific impact on the recruitment 
A

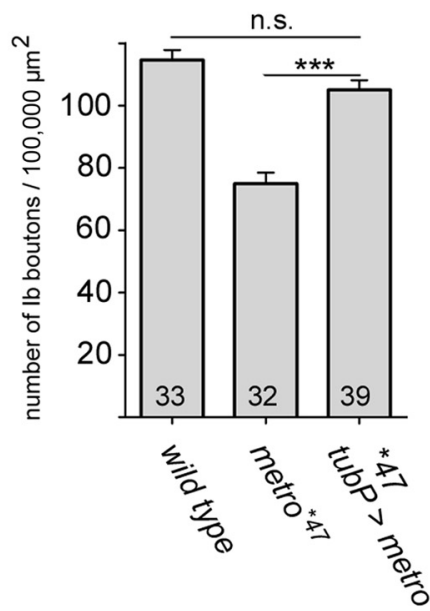

B

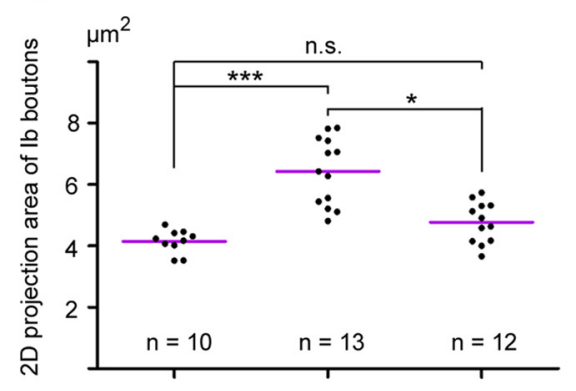

这

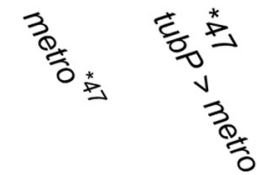

B'
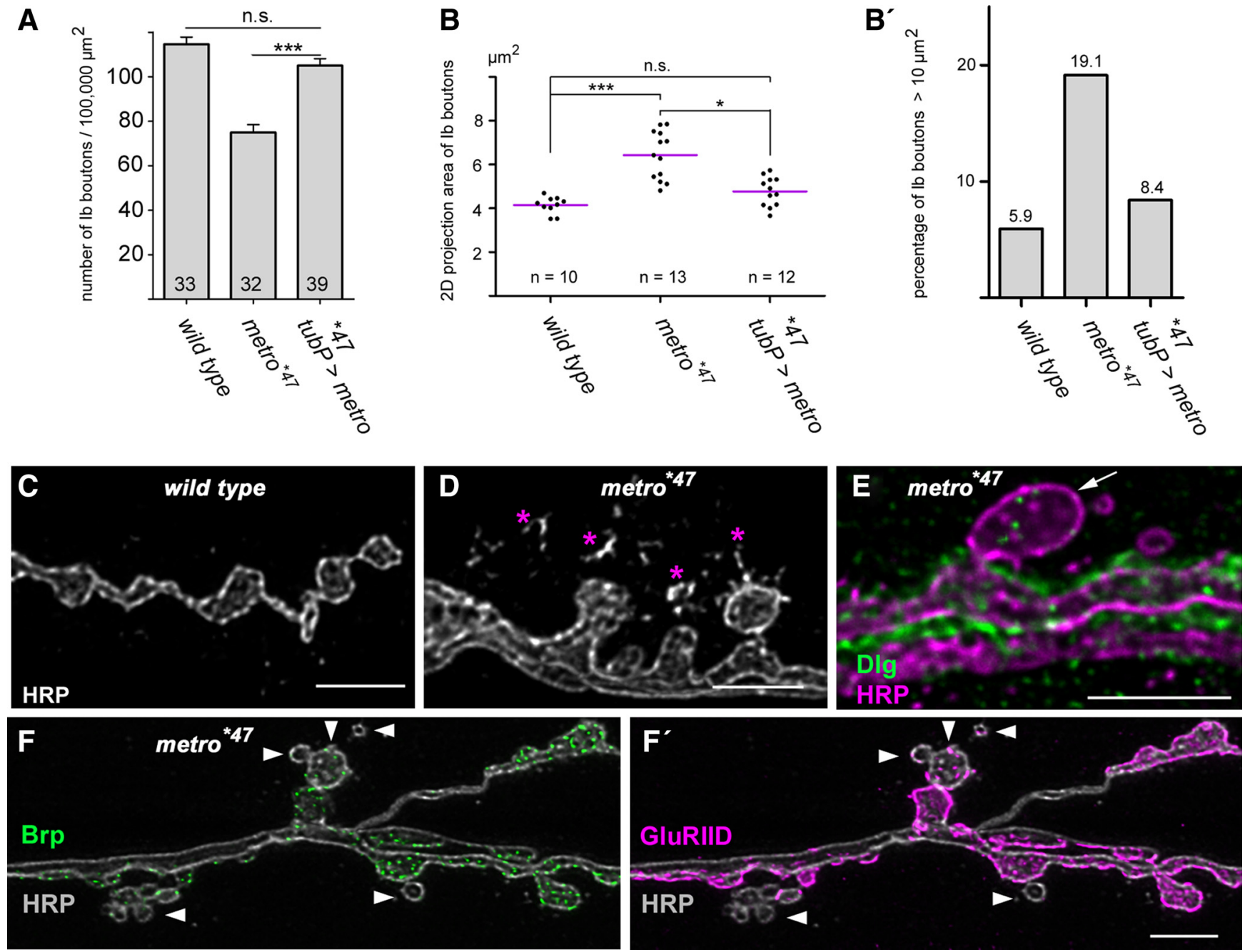

Figure 5. Metro is required for proper growth and morphology of NMJs. A, Quantification of Ib boutons on muscles $6 / 7$ of segment $A 2$, normalized to $100,000 \mu \mathrm{m}^{2}$ of muscle surface area. Wild type, $114.63 \pm 18.29$ (mean \pm SD); metro ${ }^{*} 77,75.30 \pm 20.12 ;$ metro $^{*}{ }^{47}$ plus ubiquitous Metro-B, $105.05 \pm 19.01$. Numbers of scored NMJs are indicated at the bottom of each column. Error bars correspond to SEMs. n.s., No significant difference, $p>0.05$; ${ }^{* * *}$, highly significant, $p<0.001$ ( $t$ test). $\boldsymbol{B}$, Quantification of Ib bouton projection areas. Each plotted value is a mean value derived from the analysis of boutons at a single NMJ (supplemental Table 1, available at www.jneurosci.org as supplemental material). Mean bouton areas \pm SD were as follows: wild type, $4.14 \pm 0.38$ $\mu \mathrm{m}^{2} ;$ metro $^{* 47}, 6.43 \pm 1.1 \mu \mathrm{m}^{2} ;$ metro $^{* 47}$ plus ubiquitous Metro-B, $4.77 \pm 0.67 \mu \mathrm{m}^{2}$. The statistical test was the nonparametric Kruskal-Wallis test plus Dunn's multiple comparison. n.s., Not significant, $p>0.05 ;{ }^{*} p<0.05 ;{ }^{* * *} p<0.001$. n corresponds to the number of NMJs analyzed. $\boldsymbol{B}^{\prime}$, Percentage of boutons with projection areas of $>10 \mu \mathrm{m}^{2}$. C, $\boldsymbol{D}$, anti-HRP-stained NMJ branches of wild-type $(\boldsymbol{C})$ and metro $^{*}{ }^{* 7}$ mutant $(\boldsymbol{D})$ larvae. Asterisks mark striking corpuscles typically associated with mutant NMJs. $\boldsymbol{E}-\boldsymbol{F}^{\prime}$, Boutons with impaired differentiation as indicated by the absence of $\mathrm{Dlg}\left(\boldsymbol{E}\right.$, arrow) and/or by the low abundance of Brp-and GluRIID-immunoreactive spots $\left(\boldsymbol{F}, \boldsymbol{F}^{\prime}\right.$, arrowheads) were found on virtually each metro mutant NMJ. Scale bars: $5 \mu \mathrm{m}$.

of Metro- $D$ Lin-7. These results, however, also imply that an appreciable portion of Metro- $D$ Lin-7 can associate with the NMJ independent of Dlg.

Early studies on erythrocytes have established a link between p55/MPP1 and the spectrin cytoskeleton via the protein band 4.1R. Coracle, a Drosophila band 4.1 protein, was detected at late embryonic NMJs, but no postsynaptic NMJ localization was evident at later stages (Chen et al., 2005). We therefore assessed a potential link between Metro- $D$ Lin-7 and junctional spectrin directly, through postsynaptic depletion of the latter. This was achieved by $\beta$-spectrin-specific RNAi in muscles (Pielage et al., 2006). Despite profound effects on the SSR, depletion of $\beta$-spectrin had little effect on the level of junctional $D$ Lin-7 (Fig. 4I, I'). Gal4C57-mediated expression of $\beta$-spectrin-specific RNAi in the absence of DlgS97, however, caused a reduction of junctional $D$ Lin-7 to near background levels (Fig. $4 J, J^{\prime}$ ). The spectrin-based cytoskeleton thus contributes to DlgS97-independent NMJ localization of $D$ Lin-7.

\section{Metro stabilizes the growth of NMJs}

Having established Metro as a component of the DlgS97-based scaffold complex we next aimed to specify its role at NMJs. We first assessed NMJ growth by determining the number of type $\mathrm{Ib}$ boutons normalized to the surface area of the respective muscles. Compared with the wild type, the metro ${ }^{* 47}$ mutants displayed a $35 \%$ reduction in bouton number (Fig. $5 A$ ). Ubiquitous, i.e., tubP-Gal4-driven expression of Metro-B in a metro mutant background, restored normalized bouton numbers to wild-type levels, indicating that the observed growth defect is due to loss of Metro (Fig. 5A). We also noted an elevated frequency of extralarge and irregularly shaped boutons at metro mutant NMJs. To estimate this observation we measured 2D-projected areas of anti-HRP-stained Ib boutons (supplemental Table 1, available at www.jneurosci.org as supplemental material). Mean areas of 4.14 $\mu \mathrm{m}^{2}$ for the wild type were increased by $\sim 55 \%$ in $\mathrm{metro}^{{ }^{* 47}} \mathrm{mu}$ tants (Fig. 5B). Median values were shifted to virtually the same extent, reflecting that boutons are enlarged across the entire size 

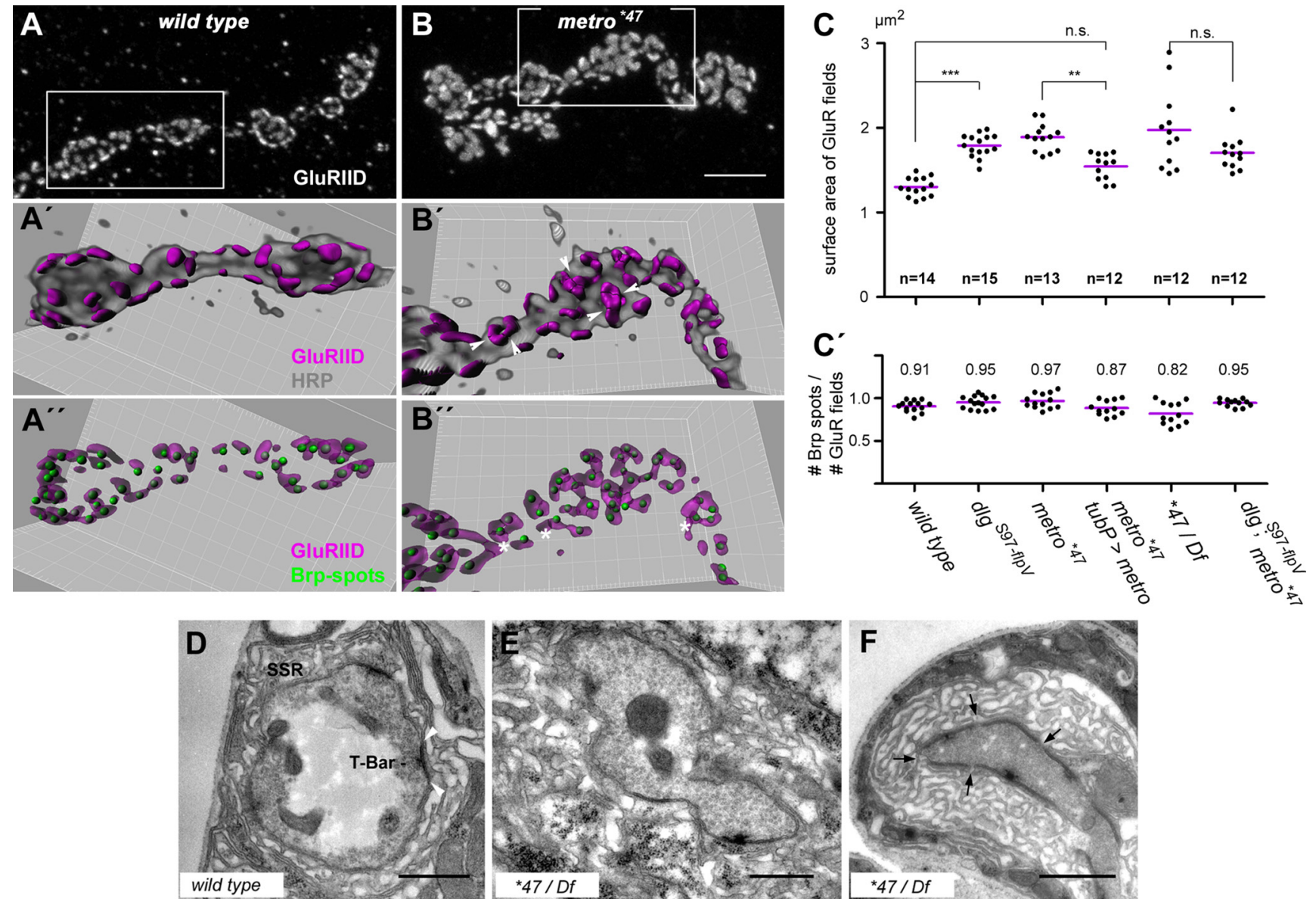

Figure 6. GluR fields are enlarged in metro mutants. $A, B$, Maximum projections of confocal stacks of wild-type $(\boldsymbol{A})$ and homozygous metro ${ }^{*}$ (B7 $(\boldsymbol{B})$ NMJ branches stained for GluRIIID. $\boldsymbol{A}^{\prime}-\boldsymbol{B}^{\prime \prime}$, Blend mode 3 D representations of GluR fields obtained by surface segmentation are shown with volume-rendered HRP labeling $\left(\boldsymbol{A}^{\prime}, \boldsymbol{B}^{\prime}\right)$ or with spot-detected Brp staining $\left(\boldsymbol{A}^{\prime \prime}, \boldsymbol{B}^{\prime \prime}\right)$. Images correspond to framed areas in $\boldsymbol{A}$ and $\boldsymbol{B}$. Note that the HRP staining hides several GluR fields due to the 3D reconstruction. Arrowheads in $\boldsymbol{B}^{\prime}$ point to boundaries of closely juxtaposed GluR fields. Occasionally, image processing may lead to inappropriate segmentation of large, irregularly shaped receptor fields, yielding fields that lack adjunct Brp spots (asterisks in $\boldsymbol{B}^{\prime \prime}$ ). Note that each Brp spot is associated with a receptor field. C, Quantification of GluR field sizes. Each of the plotted values is the mean value derived from the analysis of at least 200 receptor fields at a single NMJ and $n$ corresponds to the number of NMJs analyzed. Mean sizes \pm SD were as follows: wild type, $1.301 \pm 0.114 \mu \mathrm{m}^{2} ; \mathrm{dlg}^{\text {S97-flpV }}, 1.792 \pm 0.133 \mu \mathrm{m}^{2} ;$ metro $^{*}{ }^{* 77}, 1.888 \pm 0.160 \mu \mathrm{m}^{2} ;$ metro ${ }^{* 47}$ plus ubiquitous Metro-B, $1.545 \pm 0.154 \mu \mathrm{m}^{2} ;$ metro $^{*}{ }^{* 7} / \mathrm{Df}, 1.973 \pm 0.462 \mu \mathrm{m}^{2} ; \mathrm{dlg}^{597-f p V} ;$ metro ${ }^{*}{ }^{47}, 1.704 \pm 0.201 \mu \mathrm{m}^{2}$. n.s., Not significant, $p>0.05 ;{ }^{* *} p<0.01 ;{ }^{* * *} p<0.001$ (nonparametric Kruskal-Wallis test plus Dunn's multiple comparison). $\boldsymbol{C}^{\prime}$, Ratios between the number of Brp spots and the number of receptor fields, determined for the same samples as in $\boldsymbol{C}$. Mean values are indicated. No significant differences were detected (Kruskal-Wallis test). $\boldsymbol{D}-\boldsymbol{F}$, Electron micrographs of wild-type $(\boldsymbol{D})$ and mutant $(\boldsymbol{E}, \boldsymbol{F})$ type lb boutons. Arrowheads in $\boldsymbol{D}$ delineate an electron-dense active zone carrying a T-bar. An example of closely juxtaposed electron-dense zones is shown in $\boldsymbol{F}$ with arrows pointing to minimal spacings. Scale bars: (in $\boldsymbol{B}) \boldsymbol{A}, \boldsymbol{B}, 5 \mu \mathrm{m} ; \boldsymbol{D}-\boldsymbol{F}, 1 \mu \mathrm{m}$.

spectrum. Accordingly, the percentage of boutons with projection areas of $>10 \mu \mathrm{m}^{2}$ was clearly increased in the metro mutants (Fig. $5 B^{\prime}$ ). Rescue experiments based on ubiquitous expression of Metro- $B$ led to a significant restoration of the mean bouton size and to a reduced scattering of values (Fig. 5B). Metro thus controls the size of synaptic boutons and confers a certain level of homogeneity on top of the structural plasticity of NMJs.

A closer inspection of NMJs revealed further abnormalities associated with loss of Metro. HRP-labeling of wild-type NMJs outlines the surface of presynaptic terminals surrounded by a fine-grained staining reaching into the muscle area (Fig. 5C). Recent observations suggest that such grains represent so-called synaptopods of nerve terminal origin or remains thereof (Ataman et al., 2008). In both homozygous metro ${ }^{* 47}$ and hemizygous metro $^{* 47} / D f(2 R) E 3363$ mutants, this corpuscular aspect of the HRP staining was dramatically pronounced (Fig. $5 D$ ). Moreover, in contrast to the wild type, metro mutant NMJs always harbored some boutons with very little Dlg immunoreactivity (Fig. 5E) or with only a few spots of immunoreactivity for Brp and the glutamate receptor subunit GluRIID (Fig. 5F, $F^{\prime}$ ). This suggests that loss of Metro increases the rate of failed attempts to establish new synaptic boutons.

We next asked whether along with the aberrant NMJ morphology, the total number of synaptic contact sites might be altered at metro mutant NMJs. To this end we counted Brp-positive synaptic contacts by subjecting 3D reconstructions to a spot detection algorithm (Fig. S4, available at www.jneurosci.org as supplemental material). Remarkably, the number of synaptic contact sites, a crucial determinant of synaptic strength, was almost identical in the wild type and metro ${ }^{* 77}$ mutants $(1548 \pm 96$ versus $1472 \pm 132$ spots per $100,000 \mu \mathrm{m}^{2}$ of muscle surface area).

\section{Metro controls the size of glutamate receptor fields}

It has recently been shown that DlgS97 is required to restrict the size of synaptic contacts at NMJs (Mendoza-Topaz et al., 2008). We reasoned that Metro might be involved in this aspect of synaptic organization as a downstream effector of DlgS97. To address this issue, we established a quantitative imaging strategy, which enabled us to assess large numbers of synaptic contact sites 
at NMJs from various genotypes. This was based on 3D reconstructions from confocal stacks of NMJ branches stained for HRP, Brp, and GluRIID (Fig. 6A-C; supplemental Table 2, available at www.jneurosci.org as supplemental material). The latter is a constituent of both subtypes of ionotropic GluRs at NMJs (Qin et al., 2005) and therefore GluRIID-specific immunoreactivity basically represents entire receptor fields. Normally, synaptic contacts are quite evenly distributed over the surface of boutons, obeying a certain spacing zone (Meinertzhagen et al., 1998). Accordingly, receptor fields at wild-type NMJs occurred as well separated entities (Fig. 6 $A^{\prime}$ ), for which the algorithmic measurement yielded a mean surface area of $1.301 \pm 0.114 \mu \mathrm{m}^{2} \mathrm{SD}$ (NMJs analyzed: $n=14$ ) (Fig. $6 C$ ). It should be stressed that this value corresponds to the surface of $3 \mathrm{D}$ reconstructions of receptor fields and hence exceeds the range expected for receptor fields in terms of their planar expansion within the postsynaptic membrane (see Materials and Methods). We next determined the size of receptor fields at NMJs lacking DlgS97. Compared with the wild type, the receptor fields of the here-described $d l g$ S97-flpV allele were enlarged by $\sim 40 \%\left(1.792 \pm 0.133 \mu \mathrm{m}^{2} ; n=15\right)$ (Fig. 6C). This result is in remarkable agreement with data obtained by quantitative electron microscopy for the $d l^{597-138}$ allele (Mendoza-Topaz et al., 2008) and thus validates our approach. Compared with controls, the metro mutants often displayed large patches of GluRIID immunoreactivity (Fig. $6 \mathrm{~B}$ ). Image analysis, however, indicated that many of the patches were composed of two or more barely separated receptor fields, of which by far the most were associated with the active zone marker Brp (Fig. $6 B^{\prime}, B^{\prime \prime}$; Fig. S4, available at www.jneurosci.org as supplemental material). This result was substantiated by an electron microscopical inspection of Ib boutons (Fig. 6D-F). Synaptic contacts at mutant boutons appeared largely normal with regard to electron density and the presence of presynaptic T-bars (Fig. 6E,F). The length of individual electron dense zones observed on single sections of mutant boutons was mostly in the range of 400-800 $\mathrm{nm}$, i.e., similar to that of controls. However, we frequently observed tightly juxtaposed electron dense zones on sections of mutant boutons (Fig. 6F), consistent with the partitioning obtained by our algorithmic image analysis. Despite such partitioning, the mean surface area of receptor fields was still considerably increased in both the homozygous and hemizygous mutants $\left(1.888 \pm 0.160 \mu \mathrm{m}^{2}, n=13\right.$ and $\left.1.973 \pm 0.462 \mu \mathrm{m}^{2}, n=12\right)$. Haploinsufficiency for genes other than metro but covered by the deficiency may account for the more variable phenotype at hemizygous NMJs. Ubiquitous expression of Metro-B in the homozygous mutant background resulted in a moderate yet significant downsizing of the receptor fields $\left(1.545 \pm 0.154 \mu \mathrm{m}^{2} ; n=12\right)$ (Fig. 6C). The mean size of receptor fields at $d l g^{S 97-f l p V}$; metro ${ }^{* 47}$ double mutant NMJs $(1.704 \pm 0.201 ; n=12)$ did not significantly deviate from the single mutants (Fig. 6C). This latter finding supports the idea that DlgS97 and Metro act in the same pathway to control the size of receptor fields.

\section{Loss of Metro does not affect basal synaptic transmission}

To clarify whether loss of Metro affects synaptic transmission, we performed electrophysiological recordings from muscle 6 of hemizygous metro mutants. Both the frequency of spontaneous release of single vesicles (minifrequency) (Fig. $7 A, A^{\prime}$ ) and the amplitudes of the resulting miniature excitatory junctional currents (mEJCs) (Fig. $7 A^{\prime \prime}$ ) were indistinguishable from hemizygous controls (+/Df) and are in the range of other controls recorded previously under the same conditions (Kittel et al., 2006). The amplitude of mEJCs is commonly interpreted as the
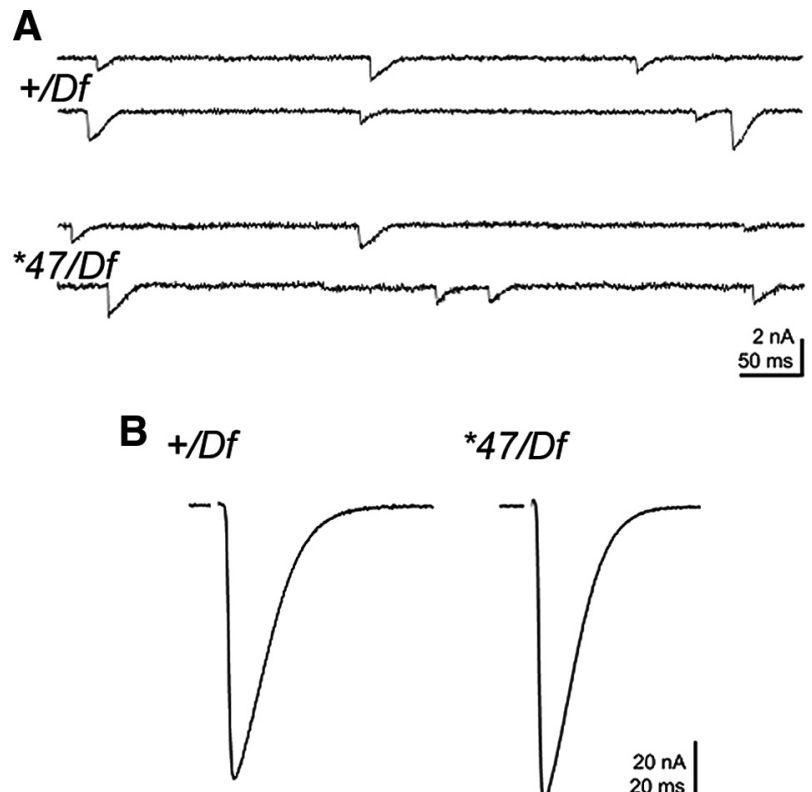

*47/Df

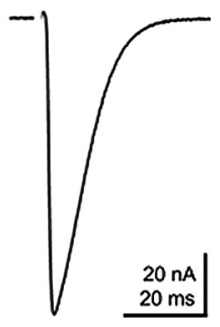

Figure 7. Basal transmission is normal at metro mutant NMJs. A, Electrophysiological recordings of spontaneous release events. No significant differences were evident between controls $(+/ D f)$ and mutants $(* 47 / D f)$ regarding the frequency $(1.25 \pm 0.31$ vs $1.19 \pm 0.10 \mathrm{~Hz}$ ) and amplitude $(-0.91 \pm 0.05$ vs $-0.83 \pm 0.03 \mathrm{nA})$ of $\mathrm{mEJCs}$. $\boldsymbol{B}$, The amplitudes of evoked responses upon $0.2 \mathrm{~Hz}$ stimulation were comparable between controls and mutants $(-75.7 \pm$ $6.1 \mathrm{vs}-86.3 \pm 4.3 \mathrm{nA}$ ), as were the number of vesicles released per action potential (quantal content; $84 \pm 7$ vs $107 \pm 8)$.

responsiveness of the postsynaptic receptor fields, which hence appears unaffected. Likewise, a slight increment in the amplitude of EJCs evoked by low-frequency stimulation of motoneurons did not reach statistical significance (Fig. $7 B, B^{\prime}$ ). Consequently, an apparent increase in quantal content (Fig. $7 B^{\prime \prime}$ ) may not be considered as statistically significant. Moreover, a tendency toward faster desensitization for mutant EJCs was not evident at the level of individual mEJCs (data not shown). We therefore conclude that despite considerable structural abnormalities, the metro mutants maintain essentially normal basal synaptic transmission.

\section{DlgS97, Metro, and DLin-7 control each other to various extents}

Our analyses so far implied that Metro acts downstream of DlgS97 at NMJs. Studies on related vertebrate scaffold complexes, however, suggested a reverse dependency in cultured epithelial cells, as proper membrane localization of SAP97 $\beta$ (hDlg) was found to rely on CASK or MPP7 (Lee et al., 2002; Bohl et al., 2007). We therefore wondered whether Metro would promote the junctional localization of Dlg. The level of total Dlg at NMJs as revealed by anti-Dlg $\operatorname{PDz}_{\mathrm{PD}}$ immunoreactivity appeared modestly reduced in metro mutants (data not shown). In contrast, junctional DlgS97 was clearly diminished (Fig. $8 A, B$ ) and quantification revealed a reduction by $\sim 43 \%$ (Fig. $8 D$ ) relative to wild-type levels. Muscle expression of Metro-B restored the abundance of DlgS97 at NMJs to near-wild-type levels (90.3\% relative to wild type) (Fig. $8 C, D$ ). These findings imply that Metro selectively enhances the anchoring of DlgS97 at NMJs.

Surprisingly, when we inspected NMJs of DLin-7-null mutants (Bachmann et al., 2008b) we noticed a reduction of DlgS97 to approximately the same extent as observed for metro mutants (Fig. $8 E, F$ ). This finding argues against $D$ Lin-7 acting purely 
downstream of DlgS97 and Metro. We therefore assessed the impact of DLin-7 on the NMJ localization of Metro. Strikingly, Metro labeling was completely absent from DLin-7 mutant NMJs (Fig. $\left.8 E^{\prime}, F^{\prime}\right)$, but was completely restored by targeted expression of Flag-DLin-7 in muscles (data not shown). RT-PCR analysis confirmed that in the absence of $D$ Lin-7 metro is still expressed at the transcriptional level (Fig. 8G). Western blot analyses, however, revealed the complete absence of Metro from larval body wall muscles (Fig. $8 \mathrm{H}$ ). As depicted in Figure $1 A$, Metro carries a high-score PEST sequence that partially overlaps with the DLin-7-binding L27C domain. We therefore speculated that $D$ Lin-7 might prevent the degradation of Metro by covering its PEST sequence. Gal4-C57-driven expression of Metro-B in the absence of DLin-7, however, resulted in expression of the protein clearly above wild-type levels (Fig. $8 H$ ). Despite this overexpression, Metro-B still failed to localize at NMJs (Fig. 8I, $I^{\prime}$ ). Thus, while degradation might account for the absence of Metro in DLin-7 mutants, DLin-7 is required in a more direct manner to ensure the localization of Metro at NMJs. We thus propose that binding of DLin-7 to Metro promotes binding of the latter to DlgS97 through an allosteric mechanism as schematized in Figure $8 \mathrm{~J}$. We further conclude that DLin-7 and Metro act in a strictly interdependent manner to stabilize DlgS97 at NMJs (Fig. $8 \mathrm{~K}$ ).

\section{DLin-7 mutant NMJs exhibit a metro-like phenotype}

Given the strict dependency of Metro on DLin-7 one should expect null mutations in DLin-7 to phenocopy loss of Metro at NMJs. In fact, we observed that both homozygous and hemizygous DLin-7 mutant NMJs constantly display signs of impaired bouton differentiation just like those observed for metro mutants (Fig. 9A,B). Compared with what is seen for the wild type, NMJ growth in terms of normalized bouton numbers was reduced by $\sim 30 \%$ in both homozygous and hemizygous DLin-7 mutants $(77.68 \pm 9.88 \mathrm{SD}, n=12$ and $81.91 \pm 16.23, n=$ 30 vs $114.63 \pm 18.29, n=33, p<0.001$ versus the wild type for both genotypes), i.e., to almost the same extent as in the metro mutants. Moreover, we found that the mean projection area of type Ib boutons was increased by $63 \%$ in hemizygous DLin-7 mutants (Fig. 9C). This phenotype was largely reverted when we expressed a UAS-DLin-7 transgene presynaptically and postsynaptically by using a combination of activator strains Gal4-OK6 and Gal4-C57 (Fig. 9C). To conclude the comparative phenotyping we eventually assessed the size of synaptic contacts at hemizygous DLin-7 mutant NMJs, again by quantitative imaging of 3D reconstructions of GluRIID-stained receptor fields. Though less pronounced than in the metro mutants the mean size
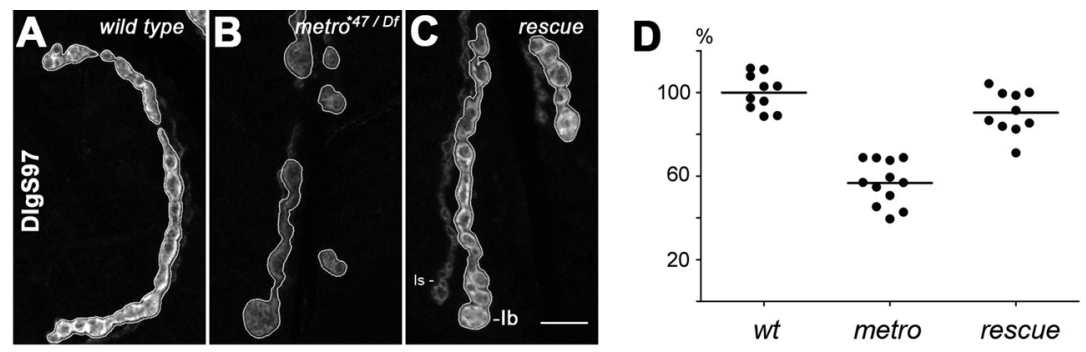

G

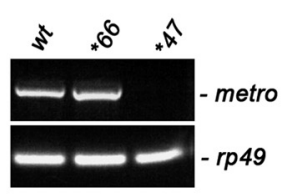

H

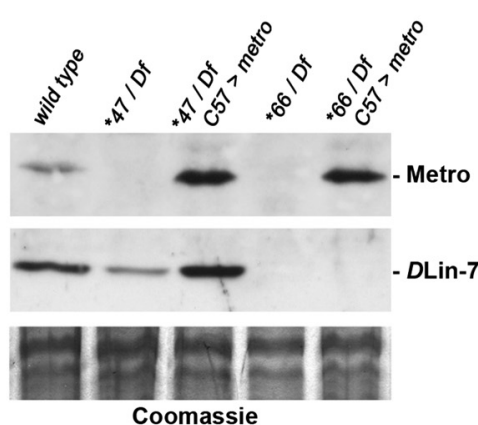

$\mathbf{K}$

DigS97 $\underset{\sim 40 \%}{\stackrel{\sim 75 \%}{\rightleftarrows}}$ Metro $\underset{100 \%}{\stackrel{100 \%}{\rightleftarrows}}$ DLin-7

Figure 8. DLin-7 controls NMJs levels of Metro and DlgS97. $\boldsymbol{A}-\boldsymbol{C}$, Reduction of junctional DlgS97 due to loss of Metro $(\boldsymbol{B})$ is of ROls as a basis for reliable measurement of fluorescence intensities. $\boldsymbol{D}$, Fluorescence intensities. Each symbol corresponds to the normalized mean value derived from measuring at least two branches of an individual NMJ. Wild type, $100 \%$ ( $\pm 8.6 \%$ SD) vs wild type). $\boldsymbol{E}-\boldsymbol{F}^{\prime}$, DLin-7 mutant NMJs exhibit reduced levels of DlgS97 $(\boldsymbol{E}, \boldsymbol{F})$ and virtually no Metro $\left(\boldsymbol{E}^{\prime}, \boldsymbol{F}^{\prime}\right) . \boldsymbol{G}, \mathrm{RT}-\mathrm{PCR}$ analysis

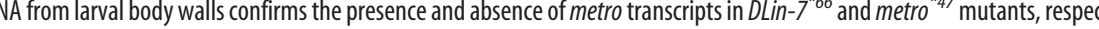
same samples were probed with Metro- and DLin-7-specific antisera. Coomassie staining served as a loading control. Note the absence of Metro in DLin-7 mutants, which can be overcome by Gal4 -C57-driven expression of UAS-metro-B. I, I', Overexpressed Metro fails to localize at NMJs in the absence of $D$ Lin-7. J, Schematic model depicting the promotional effect of $D$ Lin-7 on the binding of Metro to DlgS97. Only the L27 domains are shown. $\boldsymbol{K}$, Diagrammatic representation of the degree of interdependency between DlgS97, Metro, and DLin-7 in regard to NMJ localization. Scale bars: (in $\boldsymbol{C}, \boldsymbol{F}^{\prime}$ ), $\boldsymbol{A}-\boldsymbol{C}, \boldsymbol{E}-\boldsymbol{F}^{\prime}, 10 \mu \mathrm{m}$; (in $\boldsymbol{I}^{\prime}$ ) $\boldsymbol{I}, \boldsymbol{I}^{\prime}, 40 \mu \mathrm{m}$.

of receptor fields was significantly increased compared with the wild type (Fig. 9D). Importantly, presynaptic and postsynaptic expression of DLin-7 in the mutant background reduced the size of receptor fields to the wild-type level (Fig. 9D).

\section{Discussion}

In this study we have focused on a complex formed by Drosophila SAP97 $\beta$ alias DlgS97, the MPP-like MAGUK Metro, and the Veli/MALS homolog DLin-7. Related scaffold complexes exist at vertebrate epithelial junctions (Karnak et al., 2002; Lee et al., 2002) and at presynaptic photoreceptor terminals (Aartsen et al., 2006; Kantardzhieva et al., 2006). The existence of the respective complexes in vertebrate CNS neurons and their synaptic or extrasynaptic roles therein remain elusive. Using larval NMJs as an in vivo model, we have now shown that DlgS97-Metro-DLin-7-type complexes indeed control the proper organization of a synaptic junction. 

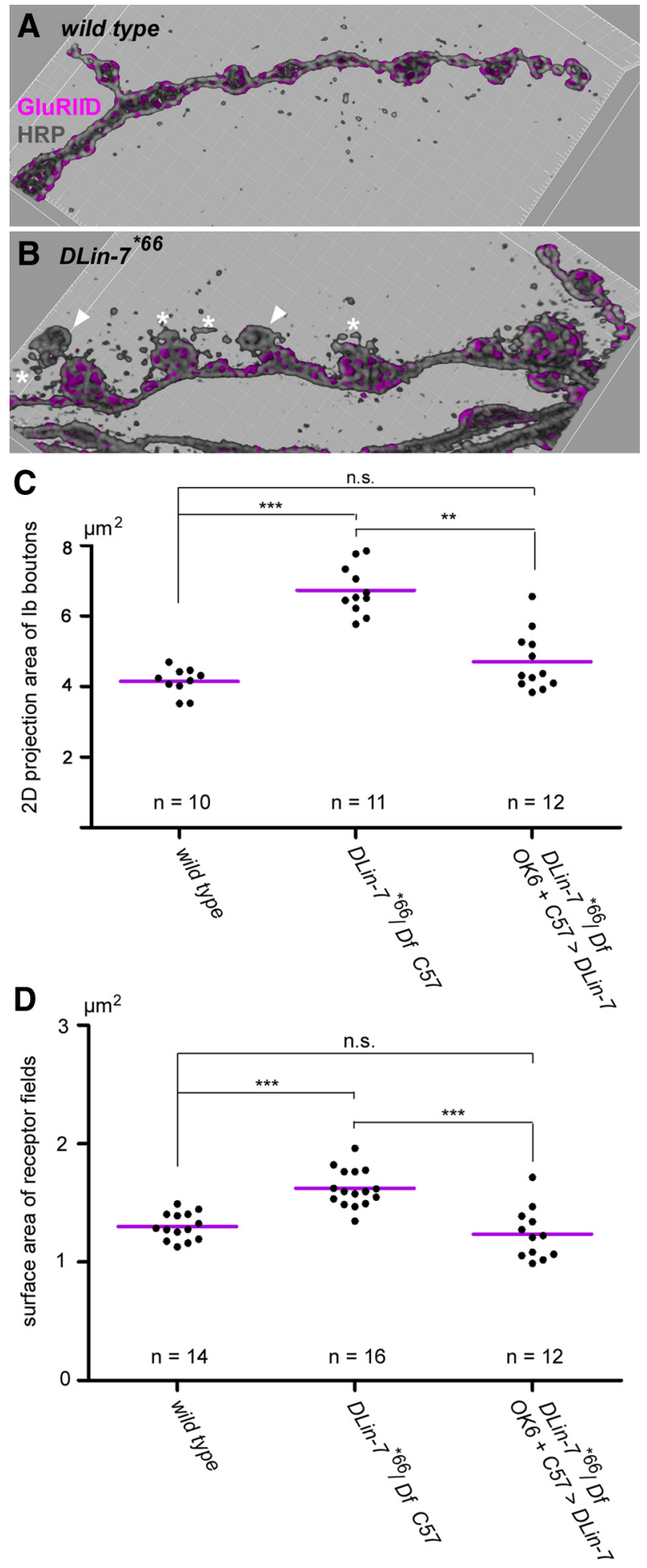

Figure 9. DLin-7 mutants show aberrant NMJ organization. $A, B$, Blend mode 3 D representations of NMJ branches from the wild type and DLin- $7^{* 66}$ mutants stained for GluRIID and HRP. Note the presence of boutons with little or no GluRIID (arrowheads) and the striking HRP-stained corpuscles (asterisks). C, Quantification of bouton projection areas. Each plotted value is a mean value derived from the analysis of type $\mathrm{lb}$ boutons at a single NMJ. Mean bouton areas \pm SD were as follows: wild type, $4.14 \pm 0.38 \mu \mathrm{m}^{2} ;$ DLin- $7^{\# 66} / \mathrm{Df}$, Gal4 -C57, $6.73 \pm 0.69 \mu \mathrm{m}^{2}$; DLin- $7^{\# 66 D f}$ plus presynaptic and postsynaptic $D$ Lin-7, $4.70 \pm 0.83 \mu \mathrm{m}^{2}$. D, Quantification of GluR field sizes. Each plotted value is a mean value derived from the analysis of at least $200 \mathrm{GluR}$ fields at a single NMJ. Mean sizes \pm SD were as follows: wild type, $1.301 \pm 0.114 \mu \mathrm{m}^{2}$; DLin-7 $7^{\sharp 66} / D f$, Gal4-C57, $1.624 \pm 0.157 \mu \mathrm{m}^{2}$; $D$ Lin- $7^{466 D f}$ plus presynaptic and postsynaptic $D$ Lin-7, $1.236 \pm 0.217 \mu \mathrm{m}^{2}$. The statistical test in $C$ and $\boldsymbol{D}$ was the nonparametric Kruskal-Wallis test plus Dunn's multiple comparison. n.s., Not significant, $p>0.05 ;{ }^{* *} p<0.01 ;{ }^{* * *} p<0.001$. $n$ always corresponds to the number of NMJs analyzed.
Our genetic analyses implied that Metro constitutes the exclusive link between DlgS97 and DLin-7 at NMJs. In vitro binding assays, however, revealed that the interaction between DlgS97 and Metro is fairly weak. This issue was eventually clarified by two observations: (1) DLin-7 is absolutely required for DlgS97dependent localization of Metro-B at NMJs, and (2) biochemical studies by Bohl et al. (2007) revealed that binding of the Metro homolog MPP7 to Veli promotes its binding to hDlg. This allosteric mechanism is thus evolutionary conserved and distinguishes Metro and its closest mammalian homologs from other MPP-like MAGUKs and Cmg/ Cask. In this way a one-to-one ratio of Metro and DLin-7 can be maintained, possibly translating into a balance of yet-to-be-identified junctional binding partners of the PDZ domains of either protein. Moreover, we found that the spectrin-based cytoskeleton to some extent assures the junctional anchorage of Metro and DLin-7 in the absence of DlgS97. This link might contribute to a regular positioning of DlgS97-based scaffold complexes dictated by a spectrindefined network (Pielage et al., 2006).

The knock-out of metro leads to a considerable reduction of DlgS97. Such chronic downregulation of DlgS97 may be partially compensated by recruitment of DlgA. Stabilization of DlgS97 by Metro and DLin-7, however, involves the formation of higherorder complexes driven by (1) the ability of L27 domains to form dimers of dimers (Feng et al., 2004; Li et al., 2004; Petrosky et al., 2005) and (2) the tandem arrangement of L27 domains in Metro. Formally, such complexes are unlimited (Fig. S2B, available at www.jneurosci.org as supplemental material). In addition to the reduced abundance of junctional DlgS97, we therefore consider the breakdown of its multimeric context as a crucial consequence of lacking Metro and DLin-7.

The formation of new boutons strongly correlates with a temporally restricted downregulation of the Dlg-based scaffold at the respective site (Mathew et al., 2002; Ataman et al., 2008). Normally, Dlg reassociates with the nascent bouton shortly thereafter and recent studies suggest that GluRs and the actin regulators dPix and Pak act upstream of Dlg in this process (Parnas et al., 2001; Albin and Davis, 2004; Schmid et al., 2006). The occurrence of relatively large boutons with only a few GluR clusters and very little Dlg in both metro and DLin-7 mutants suggests that the formation of the postsynaptic scaffold is disturbed. Interestingly, a knockdown of MPP7 was found to cause a significant delay in the establishment of epithelial tight junctions (Stucke et al., 2007). Considering the temporal aspect, we propose that Metro and DLin-7 are required to synchronize junctional expansion and scaffold assembly. Indeed we observed various other abnormalities that are consistent with a role for Metro and DLin-7 in balancing NMJ growth and stability. Reduced proliferation of boutons was accompanied by an overall enlargement of boutons, a reciprocal correlation, observed in several instances, possibly indicative of a disturbed linkage between submembranous scaffold and cytoskeletal elements (Stewart et al., 1996; Roos et al., 2000; Wan et al., 2000; Viquez et al., 2006; Besse et al., 2007). Notably, enlarged boutons may harbor more active zones, explaining the virtual invariance in the overall number of synaptic contacts at mutant versus wild-type NMJs.

Our data indicate a stabilizing role for Metro and DLin-7, possibly as part of a back-up system to cope with operational demands such as junctional plasticity. It remains elusive, however, whether both proteins are regulated to weaken or strengthen the Dlg-based scaffold. Weakening, as discussed above, may be involved in defining sites for bouton formation, but might also be a prerequisite for the de novo formation of synaptic contact sites 
on preexisting boutons (Rasse et al., 2005; Schmid et al., 2006). The striking protein instability of Metro in the absence of DLin-7 is suggestive in this regard, as it implies that upon breakdown, the complex can only be reassembled based on newly synthesized Metro. This would result in latency, which in turn could contribute to the temporal fidelity of the processes.

Given the strong dependency of Metro on DlgS97 it seemed plausible that loss of Metro would affect the size of receptor fields. We have used a novel method that, once established, allowed us to measure the size of a high number of receptor fields. In this way we found that the receptor fields were indeed enlarged at metro mutant NMJs. The specificity of this phenotype was further confirmed by transgenic rescue, which, however, remained incomplete. Although Metro-B clearly occurred as the principal isoform at NMJs, it is possible that the A- and/or C-variants are required at a low level or just temporarily to cover metro function at NMJs entirely. Compared with the enormous expansion of synaptic contacts associated with simultaneous depletion of DlgA and DlgS97 (Karunanithi et al., 2002; Mendoza-Topaz et al., 2008), the here-described effects on receptor field size appear moderate. It is conceivable, however, that the reduced spacing between synaptic contacts that we frequently observed in the mutants represents a prestage toward the fusion of neighboring contacts.

While the size of receptor fields differed markedly between metro mutants and controls, we did not notice striking differences in local GluRIID-specific fluorescence intensities (Fig. 6, compare $A$ to $B$ ). Moreover, despite the structural abnormalities, metro mutants displayed a rather normal profile of electrophysiological parameters. In particular, quantal currents were not significantly altered, consistent with the assumption that the composition and local density of GluRs remained normal and that transmitter release from a single vesicle does not saturate a normal-sized receptor field (Edwards, 2007). Notably, normal mEJC amplitudes have been measured in case of a pronounced enlargement of synaptic contacts (Kaufmann et al., 2002) and increased mEJC amplitudes in strong $d l g$ alleles have been assorted to enlarged synaptic vesicles rather than the size increment of synaptic contacts (Karunanithi et al., 2002). The fact that the frequency of spontaneous release events and the evoked transmission remained unaffected is consistent with the virtual invariance in the number of active zones facing GluR fields at mutant NMJs and further implies that the presynaptic release machinery is largely intact in the metro mutants.

To date there is little evidence for the enrichment of Metrolike MAGUKs at synapses in the mammalian CNS, whereas mammalian homologs of Dlg and DLin-7 are prominent presynaptic and postsynaptic components of excitatory synapses. Reminiscent of our observations, depletion of Veli in mice was found to cause a moderate increase in synaptic size, and yet this effect was assigned to its presynaptic interaction with liprin- $\alpha$ via Cask (Olsen et al., 2005). Notably, there is to our knowledge hitherto no report on a close link between DLG-MAGUKs and Veli at synapses of CNS neurons, despite the presence of Cask as a potential linker protein. Nevertheless an association of MPP3 with SAP97 and Veli is implied by coimmunoprecipitations from rat brain (Karnak et al., 2002). Moreover, MPP3 was found to bind to a serotonin receptor and to the CAM Necl-1/SynCAM3 at extrasynaptic sites (Kakunaga et al., 2005; Gavarini et al., 2006). Our results thus let us propose that the perisynaptic interplay of Metro, DlgS97, and DLin-7 represents a conserved mechanism that confers structural fidelity and stability on synaptic systems during development and plasticity.

\section{References}

Aartsen WM, Kantardzhieva A, Klooster J, van Rossum AG, van de Pavert SA, Versteeg I, Cardozo BN, Tonagel F, Beck SC, Tanimoto N, Seeliger MW, Wijnholds J (2006) Mpp4 recruits Psd95 and Veli3 towards the photoreceptor synapse. Hum Mol Genet 15:1291-1302.

Albin SD, Davis GW (2004) Coordinating structural and functional synapse development: postsynaptic p21-activated kinase independently specifies glutamate receptor abundance and postsynaptic morphology. J Neurosci 24:6871-6879.

Ataman B, Budnik V, Thomas U (2006) Scaffolding proteins at the Drosophila neuromuscular junction. Int Rev Neurobiol 75:181-216.

Ataman B, Ashley J, Gorczyca M, Ramachandran P, Fouquet W, Sigrist SJ, Budnik V (2008) Rapid activity-dependent modifications in synaptic structure and function require bidirectional Wnt signaling. Neuron 57:705-718.

Bachmann A, Knust E (2008) The use of P-element transposons to generate transgenic flies. Methods Mol Biol 420:61-77.

Bachmann A, Timmer M, Sierralta J, Pietrini G, Gundelfinger ED, Knust E, Thomas U (2004) Cell type-specific recruitment of Drosophila Lin-7 to distinct MAGUK-based protein complexes defines novel roles for Sdt and Dlg-S97. J Cell Sci 117:1899-1909.

Bachmann A, Draga M, Grawe F, Knust E (2008a) On the role of the MAGUK proteins encoded by Drosophila varicose during embryonic and postembryonic development. BMC Dev Biol 8:55.

Bachmann A, Grawe F, Johnson K, Knust E (2008b) Drosophila Lin-7 is a component of the Crumbs complex in epithelia and photoreceptor cells and prevents light-induced retinal degeneration. Eur J Cell Biol 87:123-136.

Bellen HJ, Budnik V (2000) The neuromuscular junction. In: Drosophila, a laboratory manual (Ashburner M, Hawley S, Sullivan B, eds). Cold Spring Harbor, NY: Cold Spring Harbor Laboratory.

Bellen HJ, Levis RW, Liao G, He Y, Carlson JW, Tsang G, Evans-Holm M, Hiesinger PR, Schulze KL, Rubin GM, Hoskins RA, Spradling AC (2004) The BDGP gene disruption project: single transposon insertions associated with $40 \%$ of Drosophila genes. Genetics 167:761-781.

Besse F, Mertel S, Kittel RJ, Wichmann C, Rasse TM, Sigrist SJ, Ephrussi A (2007) The Ig cell adhesion molecule Basigin controls compartmentalization and vesicle release at Drosophila melanogaster synapses. J Cell Biol 177:843-855.

Bohl J, Brimer N, Lyons C, Vande Pol SB (2007) The stardust family protein MPP7 forms a tripartite complex with LIN7 and DLG1 that regulates the stability and localization of DLG1 to cell junctions. J Biol Chem 282:9392-9400.

Brand AH, Perrimon N (1993) Targeted gene expression as a means of altering cell fates and generating dominant phenotypes. Development 118:401-415

Caruana G (2002) Genetic studies define MAGUK proteins as regulators of epithelial cell polarity. Int J Dev Biol 46:511-518.

Chen K, Featherstone DE (2005) Discs-large (DLG) is clustered by presynaptic innervation and regulates postsynaptic glutamate receptor subunit composition in Drosophila. BMC Biol 3:1.

Chen K, Merino C, Sigrist SJ, Featherstone DE (2005) The 4.1 protein coracle mediates subunit-selective anchoring of Drosophila glutamate receptors to the postsynaptic actin cytoskeleton. J Neurosci 25:6667-6675.

Chetkovich DM, Bunn RC, Kuo SH, Kawasaki Y, Kohwi M, Bredt DS (2002) Postsynaptic targeting of alternative postsynaptic density-95 isoforms by distinct mechanisms. J Neurosci 22:6415-6425.

Edwards RH (2007) The neurotransmitter cycle and quantal size. Neuron 55:835-858.

Ehrlich I, Klein M, Rumpel S, Malinow R (2007) PSD-95 is required for activity-driven synapse stabilization. Proc Natl Acad Sci U S A 104:41764181 .

Elias GM, Funke L, Stein V, Grant SG, Bredt DS, Nicoll RA (2006) Synapsespecific and developmentally regulated targeting of AMPA receptors by a family of MAGUK scaffolding proteins. Neuron 52:307-320.

Feng W, Long JF, Fan JS, Suetake T, Zhang M (2004) The tetrameric L27 domain complex as an organization platform for supramolecular assemblies. Nat Struct Mol Biol 11:475-480. 
Gavarini S, Bécamel C, Altier C, Lory P, Poncet J, Wijnholds J, Bockaert J, Marin P (2006) Opposite effects of PSD-95 and MPP3 PDZ proteins on serotonin 5-hydroxytryptamine2C receptor desensitization and membrane stability. Mol Biol Cell 17:4619-4631.

Goda Y, Davis GW (2003) Mechanisms of synapse assembly and disassembly. Neuron 40:243-264.

Gorczyca D, Ashley J, Speese S, Gherbesi N, Thomas U, Gundelfinger E, Gramates LS, Budnik V (2007) Postsynaptic membrane addition depends on the Discs-Large-interacting t-SNARE Gtaxin. J Neurosci 27:1033-1044.

Kakunaga S, Ikeda W, Itoh S, Deguchi-Tawarada M, Ohtsuka T, Mizoguchi A, Takai Y (2005) Nectin-like molecule-1/TSLL1/SynCAM3: a neural tissue-specific immunoglobulin-like cell-cell adhesion molecule localizing at non-junctional contact sites of presynaptic nerve terminals, axons and glia cell processes. J Cell Sci 118:1267-1277.

Kantardzhieva A, Alexeeva S, Versteeg I, Wijnholds J (2006) MPP3 is recruited to the MPP5 protein scaffold at the retinal outer limiting membrane. FEBS J 273:1152-1165.

Karnak D, Lee S, Margolis B (2002) Identification of multiple binding partners for the amino-terminal domain of synapse-associated protein 97. J Biol Chem 277:46730-46735.

Karunanithi S, Marin L, Wong K, Atwood HL (2002) Quantal size and variation determined by vesicle size in normal and mutant Drosophila glutamatergic synapses. J Neurosci 22:10267-10276.

Kaufmann N, DeProto J, Ranjan R, Wan H, Van Vactor D (2002) Drosophila liprin-alpha and the receptor phosphatase Dlar control synapse morphogenesis. Neuron 34:27-38.

Kim E, Cho KO, Rothschild A, Sheng M (1996) Heteromultimerization and NMDA receptor-clustering activity of Chapsyn-110, a member of the PSD-95 family of proteins. Neuron 17:103-113.

Kim K, Yang J, Zhong XP, Kim MH, Kim YS, Lee HW, Han S, Choi J, Han K, Seo J, Prescott SM, Topham MK, Bae YC, Koretzky G, Choi SY, Kim E (2009) Synaptic removal of diacylglycerol by DGKzeta and PSD-95 regulates dendritic spine maintenance. EMBO J 28:1170-1179.

Kittel RJ, Wichmann C, Rasse TM, Fouquet W, Schmidt M, Schmid A, Wagh DA, Pawlu C, Kellner RR, Willig KI, Hell SW, Buchner E, Heckmann M, Sigrist SJ (2006) Bruchpilot promotes active zone assembly, Ca2+ channel clustering, and vesicle release. Science 312:1051-1054.

Landgraf M, Sánchez-Soriano N, Technau GM, Urban J, Prokop A (2003) Charting the Drosophila neuropile: a strategy for the standardised characterisation of genetically amenable neurites. Dev Biol 260:207-225.

Lee S, Fan S, Makarova O, Straight S, Margolis B (2002) A novel and conserved protein-protein interaction domain of mammalian Lin-2/CASK binds and recruits SAP97 to the lateral surface of epithelia. Mol Cell Biol 22:1778-1791.

Lee T, Luo L (1999) Mosaic analysis with a repressible cell marker for studies of gene function in neuronal morphogenesis. Neuron 22:451-461.

Li Y, Karnak D, Demeler B, Margolis B, Lavie A (2004) Structural basis for L27 domain-mediated assembly of signaling and cell polarity complexes. EMBO J 23:2723-2733.

Lu CS, Hodge JJ, Mehren J, Sun XX, Griffith LC (2003) Regulation of the $\mathrm{Ca} 2+/$ CaM-responsive pool of CaMKII by scaffold-dependent autophosphorylation. Neuron 40:1185-1197.

Lukacsovich T, Asztalos Z, Awano W, Baba K, Kondo S, Niwa S, Yamamoto D (2001) Dual-tagging gene trap of novel genes in Drosophila melanogaster. Genetics 157:727-742.

Mathew D, Gramates LS, Packard M, Thomas U, Bilder D, Perrimon N, Gorczyca M, Budnik V (2002) Recruitment of scribble to the synaptic scaffolding complex requires GUK-holder, a novel DLG binding protein. Curr Biol 12:531-539.

Meinertzhagen IA, Govind CK, Stewart BA, Carter JM, Atwood HL (1998) Regulated spacing of synapses and presynaptic active zones at larval neuromuscular junctions in different genotypes of the flies Drosophila and Sarcophaga. J Comp Neurol 393:482-492.

Mendoza C, Olguín P, Lafferte G, Thomas U, Ebitsch S, Gundelfinger ED, Kukuljan M, Sierralta J (2003) Novel isoforms of Dlg are fundamental for neuronal development in Drosophila. J Neurosci 23:2093-2101.

Mendoza-Topaz C, Urra F, Barría R, Albornoz V, Ugalde D, Thomas U, Gundelfinger ED, Delgado R, Kukuljan M, Sanxaridis PD, Tsunoda S, Ceriani MF, Budnik V, Sierralta J (2008) DLGS97/SAP97 is developmentally upregulated and is required for complex adult behaviors and synapse morphology and function. J Neurosci 28:304-314.
Moyer KE, Jacobs JR (2008) Varicose: a MAGUK required for the maturation and function of Drosophila septate junctions. BMC Dev Biol 8:99.

Nagel AC, Maier D, Preiss A (2002) Green fluorescent protein as a convenient and versatile marker for studies on functional genomics in Drosophila. Dev Genes Evol 212:93-98.

Nakagawa T, Futai K, Lashuel HA, Lo I, Okamoto K, Walz T, Hayashi Y, Sheng M (2004) Quaternary structure, protein dynamics, and synaptic function of SAP97 controlled by L27 domain interactions. Neuron 44:453-467.

Olsen O, Moore KA, Fukata M, Kazuta T, Trinidad JC, Kauer FW, Streuli M, Misawa H, Burlingame AL, Nicoll RA, Bredt DS (2005) Neurotransmitter release regulated by a MALS-liprin-alpha presynaptic complex. J Cell Biol 170:1127-1134.

Parks AL, Cook KR, Belvin M, Dompe NA, Fawcett R, Huppert K, Tan LR, Winter CG, Bogart KP, Deal JE, Deal-Herr ME, Grant D, Marcinko M, Miyazaki WY, Robertson S, Shaw KJ, Tabios M, Vysotskaia V, Zhao L, Andrade RS, et al. (2004) Systematic generation of high-resolution deletion coverage of the Drosophila melanogaster genome. Nat Genet 36:288-292.

Parnas D, Haghighi AP, Fetter RD, Kim SW, Goodman CS (2001) Regulation of postsynaptic structure and protein localization by the Rho-type guanine nucleotide exchange factor dPix. Neuron 32:415-424.

Petrosky KY, Ou HD, Löhr F, Dötsch V, Lim WA (2005) A general model for preferential hetero-oligomerization of LIN-2/7 domains: mechanism underlying directed assembly of supramolecular signaling complexes. J Biol Chem 280:38528-38536.

Pielage J, Fetter RD, Davis GW (2006) A postsynaptic spectrin scaffold defines active zone size, spacing, and efficacy at the Drosophila neuromuscular junction. J Cell Biol 175:491-503.

Prokop A (2006) Organization of the efferent system and structure of neuromuscular junctions in Drosophila. Int Rev Neurobiol 75:71-90.

Qin G, Schwarz T, Kittel RJ, Schmid A, Rasse TM, Kappei D, Ponimaskin E, Heckmann M, Sigrist SJ (2005) Four different subunits are essential for expressing the synaptic glutamate receptor at neuromuscular junctions of Drosophila. J Neurosci 25:3209-3218.

Rasse TM, Fouquet W, Schmid A, Kittel RJ, Mertel S, Sigrist CB, Schmidt M, Guzman A, Merino C, Qin G, Quentin C, Madeo FF, Heckmann M, Sigrist SJ (2005) Glutamate receptor dynamics organizing synapse formation in vivo. Nat Neurosci 8:898-905.

Robertson HM, Preston CR, Phillis RW, Johnson-Schlitz DM, Benz WK, Engels WR (1988) A stable genomic source of P element transposase in Drosophila melanogaster. Genetics 118:461-470.

Roos J, Hummel T, Ng N, Klämbt C, Davis GW (2000) Drosophila Futsch regulates synaptic microtubule organization and is necessary for synaptic growth. Neuron 26:371-382.

Sans N, Racca C, Petralia RS, Wang YX, McCallum J, Wenthold RJ (2001) Synapse-associated protein 97 selectively associates with a subset of AMPA receptors early in their biosynthetic pathway. J Neurosci 21:75067516.

Schlüter OM, Xu W, Malenka RC (2006) Alternative N-terminal domains of PSD-95 and SAP97 govern activity-dependent regulation of synaptic AMPA receptor function. Neuron 51:99-111.

Schmid A, Qin G, Wichmann C, Kittel RJ, Mertel S, Fouquet W, Schmidt M, Heckmann M, Sigrist SJ (2006) Non-NMDA-type glutamate receptors are essential for maturation but not for initial assembly of synapses at Drosophila neuromuscular junctions. J Neurosci 26:11267-11277.

Steiner P, Higley MJ, Xu W, Czervionke BL, Malenka RC, Sabatini BL (2008) Destabilization of the postsynaptic density by PSD-95 serine 73 phosphorylation inhibits spine growth and synaptic plasticity. Neuron 60:788-802.

Stewart BA, Atwood HL, Renger JJ, Wang J, Wu CF (1994) Improved stability of Drosophila larval neuromuscular preparations in haemolymphlike physiological solutions. J Comp Physiol A Neuroethol Sens Neural Behav Physiol 175:179-191.

Stewart BA, Schuster CM, Goodman CS, Atwood HL (1996) Homeostasis of synaptic transmission in Drosophila with genetically altered nerve terminal morphology. J Neurosci 16:3877-3886.

Stucke VM, Timmerman E, Vandekerckhove J, Gevaert K, Hall A (2007) The MAGUK protein MPP7 binds to the polarity protein $\mathrm{hDlg} 1$ and 
facilitates epithelial tight junction formation. Mol Biol Cell 18:17441755.

Thomas U, Ebitsch S, Gorczyca M, Koh YH, Hough CD, Woods D, Gundelfinger ED, Budnik V (2000) Synaptic targeting and localization of discs-large is a stepwise process controlled by different domains of the protein. Curr Biol 10:1108-1117.

Viquez NM, Li CR, Wairkar YP, DiAntonio A (2006) The B' protein phosphatase $2 \mathrm{~A}$ regulatory subunit well-rounded regulates synaptic growth and cytoskeletal stability at the Drosophila neuromuscular junction. J Neurosci 26:9293-9303.

Wagh DA, Rasse TM, Asan E, Hofbauer A, Schwenkert I, Dürrbeck H, Buchner S, Dabauvalle MC, Schmidt M, Qin G, Wichmann C, Kittel R, Sigrist SJ, Buchner E (2006) Bruchpilot, a protein with homology to ELKS/
CAST, is required for structural integrity and function of synaptic active zones in Drosophila. Neuron 49:833-844.

Waites CL, Specht CG, Härtel K, Leal-Ortiz S, Genoux D, Li D, Drisdel RC, Jeyifous O, Cheyne JE, Green WN, Montgomery JM, Garner CC (2009) Synaptic SAP97 isoforms regulate AMPA receptor dynamics and access to presynaptic glutamate. J Neurosci 29:4332-4345.

Wan HI, DiAntonio A, Fetter RD, Bergstrom K, Strauss R, Goodman CS (2000) Highwire regulates synaptic growth in Drosophila. Neuron 26:313-329.

Wu VM, Yu MH, Paik R, Banerjee S, Liang Z, Paul SM, Bhat MA, Beitel GJ (2007) Drosophila Varicose, a member of a new subgroup of basolateral MAGUKs, is required for septate junctions and tracheal morphogenesis. Development 134:999-1009. 